\title{
FACTORS AND LOOSE HAMILTON CYCLES IN SPARSE PSEUDO-RANDOM HYPERGRAPHS
}

\author{
HIÊPP HÀN, JIE HAN, AND PATRICK MORRIS
}

\begin{abstract}
We investigate the emergence of subgraphs in sparse pseudo-random $k$-uniform hypergraphs, using the following comparatively weak notion of pseudo-randomness. A $k$-uniform hypergraph $H$ on $n$ vertices is called $(p, \alpha, \varepsilon)$-pseudo-random if for all (not necessarily disjoint) vertex subsets $A_{1}, \ldots, A_{k} \subseteq V(H)$ with $\left|A_{1}\right| \cdots\left|A_{k}\right| \geq \alpha n^{k}$ we have

$$
e\left(A_{1}, \ldots, A_{k}\right)=(1 \pm \varepsilon) p\left|A_{1}\right| \cdots\left|A_{k}\right| \text {. }
$$

For any linear $k$-uniform $F$ we provide a bound on $\alpha=\alpha(n)$ in terms of $p=p(n)$ and $F$, such that (under natural divisibility assumptions on $n$ ) any $k$-uniform $(p, \alpha, o(1))$-pseudo-random $n$-vertex hypergraph $H$ with a mild minimum vertex degree condition contains an $F$-factor. The approach also enables us to establish the existence of loose Hamilton cycles in sufficiently pseudo-random hypergraphs and, along the way, we also derive conditions which guarantee the appearance of any fixed sized subgraph. All results imply corresponding bounds for stronger notions of hypergraph pseudo-randomness such as jumbledness or large spectral gap.

As a consequence, $(p, \alpha, o(1))$-pseudo-random $k$-graphs as above contain: $(i)$ a perfect matching if $\alpha=o\left(p^{k}\right)$ and (ii) a loose Hamilton cycle if $\alpha=o\left(p^{k-1}\right)$. This extends the works of Lenz-Mubayi, and Lenz-Mubayi-Mycroft who studied the analogous problems in the dense setting.
\end{abstract}

\section{INTRODUCTION}

Pseudo-random graphs, vaguely speaking, are deterministic graphs which resemble their random counterparts in many characteristic properties. The systematic study of the topic was initiated by Andrew Thomason [55,56] who introduced a variant of the following notion of uniform edge distribution. A graph $G=(V, E)$ is called $(p, \beta)$-jumbled if for all (not necessarily disjoint) $A, B \subseteq V$ we have 1

$$
e(A, B):=|\{(a, b) \in A \times B:\{a, b\} \in E\}|=p|A||B| \pm \beta \sqrt{|A||B|} .
$$

The definition of jumbledness captures how close a graph is to having uniform edge distribution, with the parameter $\beta$ controlling the discrepancy from this paradigm. Further, $\beta$ also controls the size of subsets $A, B \subseteq V$ for which the lower bound in (1.1) becomes void, namely, once $|A||B|=o\left(\beta^{2} / p^{2}\right)$ holds. The random graph $G(n, p)$ is $(p, O(\sqrt{p n}))$-jumbled almost always, which is essentially optimal since it follows from the proof of Erdös and Spencer [19] that any graph with edge density, say, $p<0.99$ satisfies $\beta=\Omega(\sqrt{p n})$.

Date: August 11, 2021.

HH: Departamento de Matemática y Ciencia de la Computación, Universidad de Santiago de Chile, Las Sophoras 173, Santiago, Chile, hiep.han@usach.cl. Research supported by FONDECYT Regular grant 1191838.

JH: Department of Mathematics, University of Rhode Island, 5 Lippitt Road, Kingston, RI, USA, 02881, jie_han@uri.edu. Research partially supported by Simons Foundation \#630884.

PM: Institut für Mathematik, Freie Universität Berlin, Arnimallee 3, 14195 Berlin, Germany and Berlin Mathematical School, Germany, pm0041@math.fu-berlin.de. Research supported in part by a Leverhulme Trust Study Abroad Studentship (SAS-2017-052\9) and by the Deutsche Forschungsgemeinschaft (DFG, German Research Foundation) under Germany's Excellence Strategy - The Berlin Mathematics Research Center MATH+ (EXC-2046/1, project ID: 390685689).

${ }^{1}$ Throughout the paper we write $x=y \pm z$ to denote that $y-z \leq x \leq y+z$. 
One topic of great importance and popularity in the area concerns the appearance of certain subgraphs $F$ in sufficiently pseudo-random graphs $G$. Here, $F$ can be a small, fixed size graph such as a triangle, an odd cycle or a fixed size clique, or it can be a large, indeed spanning subgraph of $G$ such as a perfect matching, a Hamilton cycle, or a $K_{r}$-factor 2 . The fundamental question then concerns the degree of pseudo-randomness which ensures that $F$ is a subgraph of $G$ and we distinguish here the (dense) quasi-random case, when $p=\Omega(1)$ and $\beta=o(n)$, and the (sparse) pseudo-random case, when $p=o(1)$ and $\beta=\beta(n, p)$ is a function of $n$ and $p$. Most of the time proofs of results in the latter case can easily be modified to cover the former as well and in this sense problems concerning pseudo-randomness are typically more difficult than the corresponding ones for quasi-randomness. For the quasi-random case the subgraph containment problem is well understood [13, 38]; for the pseudo-random case, however, it turned out to be notoriously difficult already for small graphs $F$, and even more so for spanning subgraphs. Thus, while bounds exist for general graphs $F$ (see e.g. [4,33]), only few are known to be (essentially) best possible: triangles, odd cycles, perfect matchings, Hamilton cycles and triangle-factors [5, 6, 39, 48]. For further information on pseudo-random graphs and the related subgraph containment problem we refer the reader to the survey [39].

(Linear) pseudo-random hypergraphs. A $k$-uniform hypergraph, $k$-graph for short, is a pair $H=(V, E)$ with a vertex set $V=V(H)$ and an edge set $E=E(H) \subseteq\left(\begin{array}{l}V \\ k\end{array}\right)$, where $\left(\begin{array}{l}V \\ k\end{array}\right)$ denotes the set of all $k$-element subsets of $V$. Launched by Chung and Graham [14], the investigation of pseudorandom $k$-graphs is widely popular, albeit mostly restricted to the dense case due to the complexity of the matter $[1,10-12,15,25,26,32,34,42,43,50,52,57]$. There are several generalisations of (1.1) to $k$-graphs, the simplest and most natural of which is perhaps the following. A $k$-graph $H=(V, E)$ is called $(p, \beta)$-jumbled if for all (not necessarily disjoint) $A_{1}, \ldots, A_{k} \subseteq V$ we have

$$
e\left(A_{1}, \ldots, A_{k}\right)=p \prod_{i \in[k]}\left|A_{i}\right| \pm \beta \prod_{i \in[k]}\left|A_{i}\right|^{1 / 2} .
$$

where $e\left(A_{1}, \ldots, A_{k}\right)$ denotes the number of tuples $\left(a_{1}, \ldots, a_{k}\right), a_{i} \in A_{i}$, which form an edge in $H$.

Analogously to the graph setting, we separate the (dense) quasi-random case where $p=\Omega(1)$ and $\beta=o\left(n^{k / 2}\right)$, a range for which (1.2) only provides control over the edge distribution between linear size sets $A_{i}, i \in[k]$, and the (sparse) pseudo-random case where $p=o(1)$ and $\beta=\beta(n, p)$.

As discussed in detail below, questions concerning quasi-random hypergraphs, in particular the emergence of subgraphs therein, have received a lot of attention in recent years, see e.g. [15, 22, 23, 31, $32,42,44,45$. These results about quasirandom hypergraphs required considerable effort and so it is no surprise that much less is known in the sparse (pseudo-random) setting. We refer to [17, 24, 35, 36] for results concerning subgraph containment using stronger and more complicated notions of sparse pseudo-randomness, most prominently the work by Conlon, Fox, Zhao [17] reproving the Green-Tao Theorem.

Quasi-randomness and linear hypergraphs. The subgraph containment problem for (dense) quasirandom $k$-graphs, $k \geq 3$, has been an interesting topic and is a good example of how quasi-random hypergraphs can behave in a much more subtle manner than graphs. Indeed, with respect to subgraph statistics it is well known [13] that the number of labelled copies of any fixed size graph $F$ in a large quasi-random graph with edge density $p=\Omega(1)$ is roughly as expected from the random graph $G(n, p)$. For $k \geq 3$, however, Rödl noted that by a construction of [18], quasi-random $k$ graphs may not contain a single copy of, say, a $(k+1)$-clique, let alone the expected number of such copies. In contrast and somewhat surprisingly, the works of Kohayakawa et al. [32] and Conlon et al. 15] show that quasi-randomness is strongly related, indeed equivalent, to the counting property

\footnotetext{
${ }^{2}$ That is, vertex disjoint copies of the $r$-clique $K_{r}$ covering all the vertices of $G$.
} 
of linear $k$-graphs, those in which any two edges intersect in at most one vertex. More precisely, a sequence of $k$-graphs $\left(H_{n}\right)_{n \rightarrow \infty}, n=\left|V\left(H_{n}\right)\right|$, is $\left(p, o\left(n^{k / 2}\right)\right)$-jumbled (aka quasi-random) if and only if $H_{n}$ contains $p^{e_{F}} n^{v_{F}}+o\left(n^{v_{F}}\right)$ labelled copies of any linear $k$-graph $F$. Due to this reason, quasi-randomness for $k$-graphs is often referred to as linear quasi-randomness. Much research has looked into stronger notions of uniform edge distribution and stronger counting properties. Their relations have only been clarified recently and we refer to [57] (see also [1]) for further information.

With regards to spanning subgraphs in quasi-random $k$-graphs (with a mild minimum vertex degree), the situation also turned out to be more complex. Indeed, for graphs the famous Blowup Lemma [38] of Komlós, Sárközy and Szemerédi implies that a quasi-random graph with linear minimum degree contains any bounded-degree spanning subgraph. For $k$-graphs, no such universal statement is known but some natural spanning linear subgraphs have been studied. Lenz and Mubayi [44] and Lenz, Mubayi, and Mycroft [45] investigated the existence of perfect matchings, $F$ factors for linear $F$, and loose Hamilton cycles. As usual an $F$-factor in a $k$-graph $H$ is a collection of vertex disjoint copies of $F$ in $H$ which cover all of $V(H)$. Furthermore, a $k$-uniform loose cycle is a $k$-graph whose vertices can be cyclically ordered in such a way that each of its edges consists of $k$ consecutive vertices, and each edge intersects the subsequent edge (where the edge ordering is inherited by the ordering of the vertices) in exactly one vertex. We say that the $k$-graph $H$ contains a loose Hamilton cycle if it contains a loose cycle on $|V(H)|$ vertices as a subgraph.

For a $k$-graph $H$ and sets $U_{1}, \ldots, U_{k-1} \subseteq V(H)$, let $\operatorname{deg}\left(v ; U_{1}, \ldots, U_{k-1}\right)=e\left(\{v\}, U_{1}, \ldots, U_{k-1}\right)$ denote the degree of $v \in V(H)$ in $\left(U_{1}, \ldots, U_{k-1}\right)$. When $U_{i}=U$ for all $i \in[k-1] \operatorname{let} 3 \operatorname{deg}(v ; U)=$ $\operatorname{deg}\left(v ; U_{1}, \ldots, U_{k-1}\right)$ and define the minimum vertex degree of $H$ by $\delta(H)=\min _{v \in V(H)} \operatorname{deg}(v ; V(H))$. Lenz and Mubayi showed the following concerning factors in quasi-random $k$-graphs .

Theorem 1.1 (44]). For all $k \geq 2,0<c, p<1$ and all linear $k$-graphs $F$ on $v_{F}$ vertices there exists an $n_{0}$ and an $\varepsilon>0$ such that the following holds. If $H$ is a $\left(p, \varepsilon n^{k / 2}\right)$-jumbled $k$-graph on $n \in v_{F} \mathbb{N}$ vertices such that $n>n_{0}$, and $H$ has minimum vertex degree $\delta(H)>c n^{k-1}$, then $H$ contains an F-factor.

The result of Lenz, Mubayi and Mycroft concerning loose Hamilton cycles reads as follows.

Theorem $1.2(45])$. For all $k \geq 2$ and $0<c, p<1$, there exists an $n_{0}$ and an $\varepsilon>0$ such that the following holds. If $H$ is a $\left(p, \varepsilon n^{k / 2}\right)$-jumbled $k$-graph on $n \in(k-1) \mathbb{N}$ vertices such that $n>n_{0}$, and $H$ has minimum vertex degree $\delta(H)>c n^{k-1}$, then $H$ contains a loose Hamilton cycle.

We note here that the results in [44] and [45] are actually slightly stronger than stated and apply to $k$-graphs satisfying only the lower bound of the edge count in (1.2).

Sparse pseudo-random hypergraphs. In the sparse regime (aka the pseudo-random case) the notion (1.2) has been studied by Haviland and Thomason [31] and later by Friedman [22] and Friedman, Wigderson [23] in the context of hypergraph spectral gap. Concerning the emergence of spanning subgraphs in pseudo-random $k$-graphs we note that there is a wealth of literature on this topic for graphs, i.e., when $k=2$ (see, e.g., [2, 4, 28, 29, 39, 40, 48]). For higher uniformity, Krivelevich, Frieze and Loh 24] used a stronger and more complicated notion of pseudo-randomness to study packing of tight Hamilton cycles. The only result concerning (1.2) we are aware of is due to Lenz and Mubayi [44] who studied perfect matchings in sparse pseudo-random 3-graphs. Their result is stated in terms of hypergraph eigenvalues, a notion originated in the work of Friedman and Wigderson [22, 23] for regular $k$-graphs and extended to all $k$-graphs by Lenz and Mubayi

\footnotetext{
${ }^{3}$ Recall that we define $e\left(A_{1}, \ldots, A_{k}\right)$ to count labelled edges and so here any edge containing $v$ and vertices of $U$ is counted $(k-1)$ ! times in $\operatorname{deg}(v ; U)$.
} 
in [42. Given a $k$-graph $H=(V, E)$ (possibly with loops) on the vertex set $[n]=\{1, \ldots, n\}$ and the standard basis $\boldsymbol{e}_{1}, \ldots, \boldsymbol{e}_{n}$ of $W=\mathbb{R}^{n}$, define the $k$-linear form $\tau_{H}: W^{k} \rightarrow \mathbb{R}$ by

$$
\tau_{H}\left(\boldsymbol{e}_{i_{1}}, \boldsymbol{e}_{i_{2}}, \ldots, \boldsymbol{e}_{i_{k}}\right)= \begin{cases}1 & \text { if }\left\{i_{1}, \ldots, i_{k}\right\} \in E, \\ 0 & \text { otherwise }\end{cases}
$$

By multi-linearity this thus determines $\tau_{H}$. Let $J$ denote the all-one $k$-linear form, i.e., the form defined as above, but with $J\left(\boldsymbol{e}_{i_{1}}, \ldots, \boldsymbol{e}_{i_{k}}\right)=1$ for all $i_{1}, \ldots, i_{k} \in[n]$. Let $\|\cdot\|$ denote the Euclidean 2 -norm on $\mathbb{R}^{n}$ and for a $k$-linear form $\varphi: W^{k} \rightarrow \mathbb{R}$ let its spectral norm be defined as

$$
\|\varphi\|=\sup _{\left\|\boldsymbol{u}_{1}\right\|=\cdots=\left\|\boldsymbol{u}_{k}\right\|=1}\left|\varphi\left(\boldsymbol{u}_{1}, \ldots, \boldsymbol{u}_{k}\right)\right|
$$

Then the first and the second eigenvalue of $H$ are defined as

$$
\lambda_{1}(H)=\left\|\tau_{H}\right\| \quad \text { and } \quad \lambda(H)=\left\|\tau_{H}-\frac{k ! e(H)}{n^{k}} J\right\| .
$$

It was shown that $k$-graphs (23] for regular and [42] for all $H$ ) with second eigenvalue $\lambda=\lambda(H)$ are $(p, \lambda)$-jumbled with $p=\frac{k ! e(H)}{n^{k}}$. The following is the result by Lenz and Mubayi, which ours will improve upon in several aspects, see Theorem 1.4 and the discussion thereafter.

Theorem 1.3 ([44]). For all $0<c<1$ there exists an $n_{0}$ and an $\varepsilon>0$ such that the following holds for all $n<n_{0}$. Let $H$ be a 3 -graph on $n \in 3 \mathbb{N}$ vertices, with edge density $p:=\frac{6|E(H)|}{n^{3}}$, minimum co-degree屯 $\delta_{2}(H)>c p n^{2}$ and second eigenvalue

$$
\lambda(H) \leq \varepsilon p^{16} n^{3 / 2} .
$$

Then $H$ contains a perfect matching.

Main results. Our results rely on the following weaker version of (1.2). Given a $k$-graph $H$ and $p, \alpha, \varepsilon \in[0,1]$ we say that $H$ is $(p, \alpha, \varepsilon)$-pseudo-random if for all (not necessarily disjoint) subsets $A_{1}, \ldots, A_{k} \subseteq V(H)$ with $\left|A_{1}\right| \cdots\left|A_{k}\right| \geq \alpha|V|^{k}$ we have

$$
e\left(A_{1}, \ldots, A_{k}\right)=(1 \pm \varepsilon) p \cdot\left|A_{1}\right| \cdots\left|A_{k}\right| .
$$

Our reasons to use this notion of pseudo-randomness are twofold. Firstly, it is weaker than (1.2) and the notion of spectral gap studied by Friedman and Wigderson [22,23] and Lenz and Mubayi [42]. Indeed, note that for any $\varepsilon>0$, a $(p, \beta)$-jumbled $k$-graph (and hence $k$-graphs with density $p$ and second eigenvalue $\lambda(H) \leq \beta)$ is $(p, \alpha, \varepsilon)$-pseudo-random with $\alpha=\frac{\beta^{2}}{\varepsilon^{2} p^{2} n^{k}}$. Secondly, we believe that this notion is the most natural for this work and free from unnecessary intricacies. Indeed, in what follows it will be sufficient to have $\varepsilon>0$, which controls the variation from the average density, to be a sufficiently small constant. The parameter $\alpha=\alpha(n, p)$ then controls the size of the vertex sets for which the condition is non-trivial. When investigating subgraphs of pseudo-random (hyper-)graphs, this is often the parameter which is at the crux of the proofs, forcing the degree of pseudo-randomness necessary.

Regarding the emergence of a perfect matching in sufficiently pseudo-random hypergraphs we show the following.

Theorem 1.4. For all integers $k \geq 3$ and $c>0$ there is an $\varepsilon>0$ and an $n_{0}$ such that for any $n>n_{0}$, any $\left(p, \varepsilon p^{k}, \varepsilon\right)$-pseudo-random $k$-graph on $n \in k \mathbb{N}$ vertices with $\delta(H) \geq$ cpn $^{k-1}$ contains a perfect matching.

In particular, this holds if $H$ satisfies the minimum degree condition and is $(p, \beta)$-jumbled or is of density $p$ and has second eigenvalue $\lambda(H) \leq \beta$ with $\beta<\varepsilon p^{k / 2+1} n^{k / 2}$.

\footnotetext{
${ }^{4}$ That is, every pair of vertices in $V(H)$ is contained in at least $\delta_{2}(H)$ edges.
} 
Theorem 1.4 is a direct consequence of Theorem 1.7 from below which addresses the existence of $F$-factors for a linear $F$. Theorem 1.4 improves upon Theorem 1.3 for 3-graphs, and extends it to $k$-graphs. Indeed, Theorem 1.3 relies on the stronger notion of pseudo-randomness coming from the second eigenvalue of hypergraphs and requires that $\lambda=o\left(p^{16} n^{3 / 2}\right)$, whilst ours only requires $\lambda(H)=o\left(p^{5 / 2} n^{3 / 2}\right)$. Moreover, our result only relies on minimum vertex degree, whilst Theorem 1.3 requires minimum co-degree condition, concretely, that all pairs of vertices in $H$ are contained in $\Omega(p n)$ edges, which is a rather strong restriction in general. Indeed, while pseudo-randomness implies that most vertices have high degree, making the minimum vertex degree the natural one to consider in this context, it is easy to construct pseudo-random hypergraphs with a substantial proportion of pairs of vertices having co-degree zero.

Our approach also covers the case of loose Hamilton cycles and thus extends Theorem 1.2 to sparse pseudo-random $k$-graphs as follows.

Theorem 1.5. For any given integer $k \geq 3$ and $c>0$ there is an $\varepsilon>0$ and an $n_{0}$ such that for every $n>n_{0}$ the following holds. Suppose that $H$ is a $\left(p, \varepsilon p^{k-1}, \varepsilon\right)$-pseudo-random $k$-graph on $n \in(k-1) \mathbb{N}$ vertices with $\delta(H) \geq c p n^{k-1}$. Then $H$ contains a loose Hamilton cycle.

In particular, this holds if $H$ satisfies the minimum degree condition and is $(p, \beta)$-jumbled or is of density $p$ and has second eigenvalue $\lambda(H) \leq \beta$ with $\beta<\varepsilon p^{(k+1) / 2} n^{k / 2}$.

Finally we generalize Theorem 1.4 and address the appearance of $F$-factors in sufficiently pseudorandom $k$-graphs for any constant size linear $k$-graph $F$. It should be noted that even the appearance of a single copy of $F$ has not been explicitly studied for the sparse case, neither for (1.2) nor for (1.3). Nevertheless, we will show that the argument by Kohayakawa et al. [32] for (dense) quasi-random $k$-graphs can be extended to the sparse range, i.e., that sufficiently pseudo-random $k$-graphs contain the "expected" number of copies of any constant size, linear $k$-graph $F$.

But which degree of pseudo-randomness is sufficient for a given linear $k$-graph $F$ ? Which may be the crucial parameter(s) of $F$ which determine(s)/affect(s) the pseudo-randomness needed? Even in the case of graphs the former question is notoriously difficult and wide open, being resolved for a handful of specific graphs $F$ only. While upper bounds on the pseudo-randomness required do exist and are believed to be tight in many cases, matching lower bound constructions are rare and difficult. A common strategy to find a copy of $F$ in pseudo-random graphs relies on sequential embedding of its vertices, see e.g. [4,33, 37]. Hence the (vertex) degeneracy of $F$, i.e., $d_{F}=\max \left\{\delta\left(F^{\prime}\right): F^{\prime} \subset F\right\}$, the largest minimum degree over all induced subgraphs of $F$, is usually a crucial parameter in determining the pseudo-randomness required. We refer to [4,33, 37] for some results in similar settings in graphs which involve the vertex degeneracy (or its trivial upper bound, the maximum degree) or related notions of degeneracies [3, 16].

Unfortunately, vertex embedding strategies fail when dealing with higher uniformities and this is probably one of the main reasons why the notion of linear quasi-randomness remained obscure for a long time. As it turns out, sequential edge embedding is a suitable strategy in this setting, making the following notion of edge degeneracy a natural parameter to consider.

Briefly speaking the edge degeneracy of a linear $k$-graph $F$ is simply the vertex degeneracy of its line graph, i.e., the graph on the vertex set $E(F)$ in which two distinct vertices $e, e^{\prime} \in E(F)$ are connected if they intersect. Let us expand on this notion! For an edge $e$ in $F$ we denote by $\operatorname{deg}(e)$ its degree in the line graph of $F$. Thus $\operatorname{deg}(e)=\sum_{v \in e}\left(\operatorname{deg}_{F}(v)-1\right)$ which is equal to the number of edges $e^{\prime} \neq e$ in $F$ which intersect $e$. Let $\delta^{\prime}(F)=\min _{e \in F} \operatorname{deg}(e)$ and $\Delta^{\prime}(F)=\max _{e \in F} \operatorname{deg}(e) \operatorname{denote}$ the minimum and the maximum edge-degree of $F$, respectively. Similar to the vertex degeneracy, the edge degeneracy of $F$ can be defined as

$$
\operatorname{degen}(F)=\max \left\{\delta^{\prime}\left(F^{\prime}\right): F^{\prime} \subset F, V\left(F^{\prime}\right)=V(F)\right\},
$$


the largest minimum edge-degree taken over all subgraphs $F^{\prime} \subset F$ on the vertex set $V(F)$. There are further similarities between this notion of degeneracy and the vertex degeneracy. For example, it is easily seen that $\operatorname{degen}(F)$ is the minimum $d$ for which there is an ordering $e_{1}, \ldots, e_{s}$ of the edges of $F(s=|E(F)|)$ such that each $e_{i}$ has edge-degree at most $d$ in the spanning subgraph of $F$ induced by the edges $e_{1}, \ldots, e_{i}$. Moreover, the edge degeneracy is at most the maximum edge-degree, degen $(F) \leq \Delta^{\prime}(F)$.

As a consequence of one of our auxiliary results, Lemma 2.1, we obtain the following concerning the appearance of a single copy of a fixed size linear $k$-graph.

Theorem 1.6. For any linear $k$-graph $F$, there exists an $\varepsilon>0$ and an $n_{0}$ such that for every $n>n_{0}$, any $n$-vertex $\left(p, \varepsilon p^{\operatorname{degen}(F)}, \varepsilon\right)$-pseudo-random $k$-graph $H$ contains a copy of $F$.

In particular, the same conclusion holds if $H$ is $(p, \beta)$-jumbled or is of density $p$ and has second eigenvalue $\lambda(H) \leq \beta$ with $\beta<\varepsilon p^{\operatorname{degen}(F) / 2+1} n^{k / 2}$.

We will in fact show that one has roughly the correct number of copies of $F$ under the same pseudo-random conditions. The pseudo-randomness condition in Theorem 1.6 is known to be tight for graph triangles and conjectured to be tight for graph cliques and therefore cannot be improved in general. Indeed, for graph triangles we have $\operatorname{degen}\left(K_{3}\right)=2$ and by Alon's construction [5] there are $K_{3}$-free $n$-vertex $d$-regular graphs with $d=\Omega\left(n^{2 / 3}\right)$, which are $(d / n, \lambda)$-jumbled with $\lambda=O\left(n^{1 / 3}\right)$.

Moving on to the appearance of $F$-factors in sufficiently pseudo-random $k$-graphs our result establishes the following.

Theorem 1.7. For given integers $f \geq k \geq 2$ and $c>0$ there is an $\varepsilon>0$ and an $n_{0}$ such that for every $n>n_{0}$ the following holds. Let $F$ be a linear $k$-graph on $f$ vertices and let

$$
\ell:=\operatorname{degen}(F)+\Delta^{\prime}(F)+k .
$$

Suppose $H$ is a $\left(p, \varepsilon p^{\ell}, \varepsilon\right)$-pseudo-random $k$-graph on $n \in f \mathbb{N}$ vertices with $\delta(H) \geq c p n^{k-1}$. Then there is an $F$-factor of $H$.

In particular, this holds if $H$ satisfies the minimum degree condition and is $(p, \beta)$-jumbled or is of density $p$ and has second eigenvalue $\lambda(H) \leq \beta$ with $\beta<\varepsilon p^{\ell / 2+1} n^{k / 2}$.

Note that in $\left(p, \varepsilon p^{\ell}, \varepsilon\right)$-pseudo-random $k$-graphs, with $\ell=\operatorname{degen}(F)$ we obtain a copy of $F$ by Theorem 1.6, and with $\ell=\operatorname{degen}(F)+\Delta^{\prime}(F)+k$ we get an $F$-factor by Theorem 1.7, Recall that degen $(F) \leq \Delta^{\prime}(F)$, thus $\ell \leq 2 \Delta^{\prime}(F)+k$ which may serve as a simple upper bound on $\ell$. By taking $F$ to be a single edge we have $\Delta^{\prime}(F)=0$, thus Theorem 1.7 implies Theorem 1.4 concerning perfect matchings. In general, Theorem 1.7 extends Theorem 1.1 from dense quasi-random $k$-graphs to sparse pseudo-random $k$-graphs and establishes the first explicit condition on pseudo-randomness that guarantees the existence of a factor.

It is difficult to comment on the tightness of our general results. Indeed, at this stage it is not even clear what to conjecture as the threshold for the appearance of subgraphs of pseudorandom hypergraphs. For graphs it is believed that the obstruction for finding an $F$-factor in a pseudo-random graph is in fact the appearance of a single copy of $F$. That is, essentially the same pseudo-random conditions that guarantee a single copy of $F$ actually guarantee an $F$-factor. This intuition is confirmed only for the case $F=K_{3}$ where there still remains a log factor between the upper and lower bounds [5, 48] and in general finding $F$-free pseudo-random graphs is a very challenging problem, see e.g. [8].

One may conjecture that the same phenomenon occurs in hypergraphs, i.e., that the threshold for the appearance of a single copy of a linear $k$-graph $F$ and that for the appearance of $F$-factors coincide. In this respect Theorem 1.6 provides conditions for the appearance of a fixed linear hypergraph and so may serve as a benchmark for future work. 
Any further results providing constructions or conditions for finding general subgraphs, even for constant sized subgraphs of hypergraphs, would be very interesting. Given the generality of Theorem 1.6 and also Theorem 1.7, it will not too hard to to improve on the conditions for particular linear $k$-graphs $F$.

1.1. Proof overview and organisation. As mentioned above, Theorem 1.4 is a consequence of Theorem [1.7. The proofs of Theorem 1.7 and Theorem 1.5 work by absorption, a method popularised by Rödl, Ruciński and Szemerédi, see e.g. [53]. Both proofs follow the same scheme and so we will deal with them together. In the following we give a brief outline, ignoring some technical details.

The main step is to show that a sufficiently pseudo-random $H=(V, E)$ contains an absorbing set $A \subset V$ as follows: there is a flexible set $Z \subset A$ and an integer $m=\Omega(n)$ so that

$F$-factors: for any $Z^{\prime} \subset Z$ of size $m$ the induced $k$-graph $H\left[A \backslash Z^{\prime}\right]$ contains an $F$-factor, Ham-cyc: for any $Z^{\prime} \subset Z$ of size $m$ the induced $k$-graph $H\left[A \backslash Z^{\prime}\right]$ contains a spanning loose path with some fixed end vertices $a_{1}$ and $a_{2}$ independent of $Z^{\prime}$.

Considering the remaining vertices $V \backslash A$ and with the flexibility in mind, we find some $Z^{\prime} \subset Z$ of size $m$ so that $H\left[(V \backslash A) \cup Z^{\prime}\right]$ can be covered with disjoint $F$-copies or a spanning loose path with end vertices $a_{1}$ and $a_{2}$, respectively. The flexibility property of $Z$ then implies that $H$ contains an $F$-factor or a loose Hamilton cycle, respectively.

The absorbing set $A$ is obtained as the vertex set of an absorbing structure $\mathcal{A}$ in $H$, which is a family of copies of special $k$-graphs called absorbers. These absorbers and their properties can be found in Section 3, see Lemma 3.1 for $F$-factors and Lemma 3.2 for loose Hamilton cycles. To ensure the absorbing property of $A=V(\mathcal{A})$, the copies in $\mathcal{A}$ are not disjoint but overlap according to a certain prescribed structure called a template (see Lemma 3.4), a concept introduced by Montgomery [46, 47]. This approach requires that we deal with rooted copies of absorbers, i.e., copies in which the overlapped (aka root) vertices are pre-embedded. In Lemma 2.1 from Section 2 we show how to find such rooted copies in sufficiently pseudo-random hosts and in Lemma 2.3 we show how to put many of them together while controlling the intersection structure of the root vertices. When the intersection structure is a suitable template this lemma yields the absorbing structure $\mathcal{A}$, but it will also be useful in other steps of the proof.

In Section 4 we prove Theorem 1.7 and Theorem 1.5 both together. We close the section with the following.

Notation and properties of pseudo-random hypergraphs. Throughout the paper we omit floor and ceiling signs where they do not affect the arguments. Further, we write $\alpha \ll \beta \ll \gamma$ to mean that it is possible to choose the positive constants $\alpha, \beta, \gamma$ from right to left. More precisely, there are increasing functions $f$ and $g$ such that, given $\gamma$, whenever we choose some $\beta<f(\gamma)$ and $\alpha<g(\beta)$, the subsequent statements hold. Hierarchies of other lengths are defined similarly.

As previously mentioned, we write $x=y \pm z$ to denote that $y-z \leq x \leq y+z$. We also write equations such as $y_{1} \pm z_{1}=y_{2} \pm z_{2}$ which means that $y_{1}+z_{1} \leq y_{2}+z_{2}$ and $y_{1}-z_{1} \geq y_{2}-z_{2}$. Thus if $x=y_{1} \pm z_{1}$ and $y_{1} \pm z_{1}=y_{2} \pm z_{2}$ then $x=y_{2} \pm z_{2}$. More involved equations using \pm should all be read the same; if we turn all the \pm signs to + , then the equation holds if we replace all the $=$ signs with $\leq$ and if we turn all the \pm signs to - , the equations hold with $\geq$ signs replacing the $=$ signs. Next, we collect some easy consequences of the definition of pseudo-randomness (1.3).

Fact 1.8. Given a $(p, \alpha, \varepsilon)$-pseudo-random hypergraph $H$ and $U, V_{1}, \ldots, V_{k} \subseteq V(H)$;

- For any $\gamma>0$, if $|U| \geq \gamma^{\frac{1}{k}}|V|$, then $H[U]$ is $(p, \alpha / \gamma, \varepsilon)$-pseudo-random.

- If $\left|V_{1}\right| \cdots\left|V_{k}\right|<\alpha|V|^{k}$, then

$$
\left|e\left(V_{1}, \ldots, V_{k}\right)-p \cdot\right| V_{1}|\cdots| V_{k}|| \leq(1+\varepsilon) p \cdot \alpha|V|^{k} .
$$


Proof. The first property follows directly from the definition. For the second we extend each $V_{i}$ to a $U_{i} \subset V$ so that $\left|U_{1}\right| \cdots\left|U_{k}\right|=\alpha|V|^{k}$. Then pseudo-randomness yields

$$
0 \leq e\left(V_{1}, \ldots, V_{k}\right) \leq e\left(U_{1}, \ldots, U_{k}\right) \leq(1+\varepsilon) p \cdot \alpha|V|^{k},
$$

and the second property follows when $e\left(V_{1}, \ldots, V_{k}\right) \geq p \cdot\left|V_{1}\right| \cdots\left|V_{k}\right|$. When $p \cdot\left|V_{1}\right| \cdots\left|V_{k}\right|>$ $e\left(V_{1}, \ldots, V_{k}\right)$ the second property is immediate.

Our next lemma shows that a pseudo-random hypergraph cannot be too sparse.

Lemma 1.9. Let $\varepsilon>0$ and $H$ be $\left(p, \varepsilon p^{\ell}, \varepsilon^{\prime}\right)$-pseudo-random $k$-graph such that $\varepsilon^{\prime} \leq 1 / 2$. Then $p=\Omega\left(n^{-s}\right)$, where $s:=\frac{k(k-1)}{\ell(k-1)+k}$. In particular, if $\ell \geq k-1$, then $p=\omega(\log n / n)$.

Proof. Extending Turán's theorem to $k$-graphs, Spencer [54] (see also [7, p. 434]) showed that any $k$-graph with average (vertex-)degree $d$ has an independent set of size $c n d^{-1 /(k-1)}$ for some $c=c(k)>0$. As $e(H) \leq p n^{k}+\varepsilon^{\prime} p n^{k} \leq 2 p n^{k}$ we infer that $H$ has an independent set $I$ of size $c n\left(2 k p n^{k-1}\right)^{-1 /(k-1)}=c^{\prime} p^{-\frac{1}{k-1}}$ for some $c^{\prime}=c^{\prime}(k)>0$. Clearly $e(I, \ldots, I)=0$, yet, if $|I|^{k} \geq \varepsilon p^{\ell} n^{k}$ then pseudo-randomness implies $e(I, \ldots, I) \geq p|I|^{k}\left(1-\varepsilon^{\prime}\right)>0$, which is a contradiction. Thus, $\varepsilon p^{\ell} n^{k}>|I|^{k} \geq\left(c^{\prime} p^{-\frac{1}{k-1}}\right)^{k}$ which then yields $p=\Omega\left(n^{-s}\right)$.

\section{Finding SMALL SUBGRAPHS IN PSEUdO-RANDOM HYPERGRAPHS}

A rooted $k$-graph is a pair $(F, \mathcal{X})$ with a $k$-graph $F$ on a vertex set $V(F)=\left\{x_{1}, \ldots, x_{r}, u_{1}, \ldots, u_{f}\right\}$ and the (possibly empty) tuple $\mathcal{X}=\left(x_{1}, \ldots, x_{r}\right)$ of specified vertices such that for every two vertices $x_{i}, x_{j}$ of $\mathcal{X}$ any edge containing $x_{i}$ is disjoint from any edge containing $x_{j}$. Vertices in $\mathcal{X}$ are called roots of $(F, \mathcal{X})$ (or simply of $F$ ). Our aim is to find "rooted copies" of $(F, \mathcal{X})$ in a "sufficiently" pseudo-random $H$.

Formally, let $H$ be a $k$-graph with specified vertices $\left(y_{1}, \ldots, y_{r}\right)=\mathcal{Y}$ and $U \subseteq V(H)$ a vertex subset. A rooted copy of $(F, \mathcal{X})$ in $(H, \mathcal{Y}, U)$ (or simply of $F$ in $H)$ is an (edge preserving) embedding $\varphi: V(F) \rightarrow V(H)$ such that $\varphi\left(x_{i}\right)=y_{i}$ for all $i \in[r]$ and $\varphi\left(u_{i}\right) \in U$ for all $i \in[f]$.

To deal with rooted $k$-graphs we need to extend our notion of edge degeneracy. Recall that the edge degeneracy degen $(F)$, for a linear $k$-graph $F$, is the vertex degeneracy of its line graph. Equivalently, let $\operatorname{deg}(e)=\sum_{v \in e}(\operatorname{deg}(v)-1)$ be the degree of $e$ in the line graph of $F$, which is equal to the number of edges $e^{\prime} \neq e$ in $F$ which intersect $e$. Then $\operatorname{degen}(F)$ is the minimum $d$ for which there is an edge exposure, i.e., a permutation $\sigma \in S_{s}$ of the edge set $E(F)=\left\{e_{1}, \ldots, e_{s}\right\}$, such that each $e_{\sigma(i)}$ has degree at most $d$ in the subgraph of $F$ on the vertex set $V(F)$ and with the edges $e_{\sigma(1)}, \ldots, e_{\sigma(i)}$. For a rooted $(F, \mathcal{X})$ we additionally require from the edge exposure $\sigma$ from above that all edges containing a root appear before edges not containing any root, i.e., there are no $i>j$ such that $e_{\sigma(i)}$ contains a root and $e_{\sigma(j)}$ does not. At times, we will write $\operatorname{degen}(F, \mathcal{X})$ to make it clear that we refer to the rooted graph and hence only consider these restricted edge exposures when calculating the degeneracy. However we will also simply write $\operatorname{degen}(F)$ if $\mathcal{X}$ is clear from context.

2.1. A counting lemma for rooted $k$-graphs. When $H$ is sufficiently pseudo-random with respect to a fixed rooted graph $F$, and satisfies certain mild degree conditions, then the following lemma guarantees many rooted copies of $F$ in $H$. It is an extension of an argument by Kohayakawa et al. [32] to the sparse case.

Lemma 2.1 (Rooted counting). For integers $k, f \geq 2, r \geq 0$ and $1 \geq c>0$ there is an $\varepsilon>0$ and an $n_{0} \in N$ such that the following holds for all $n \geq n_{0}$. Let $\left(F,\left(x_{1}, \ldots, x_{r}\right)\right)$ be a rooted linear $k$-graph on $r+f$ vertices and with edge degeneracy $\ell$. Suppose that $H$ is a $\left(p, \varepsilon p^{\ell}, \varepsilon\right)$-pseudo-random $k$-graph 
on $n$ vertices with $\Delta_{2}(H)<\varepsilon p n^{k-1}, U \subseteq V(H)$ a set of size $|U| \geq$ cn and $y_{1}, \ldots, y_{r} \in V(H)$ vertices, which satisfy $\operatorname{deg}\left(y_{i} ; U\right) \geq c p|U|^{k-1}$ for each $i \in[r]$. Then there are at least

$$
\frac{1}{2}(c p)^{e(F)}|U|^{f}
$$

rooted copies of $\left(F,\left(x_{1}, \ldots, x_{r}\right)\right)$ in $\left(H,\left(y_{1}, \ldots, y_{r}\right), U\right)$.

Note that $\Delta_{2}(H)<\varepsilon p n^{k-1}$ is a rather weak condition, which moreover can be dropped if $\ell \geq k-1$. Indeed, in this case $p \gg 1 / n$ by Lemma 1.9 and thus $\Delta_{2}(H) \leq n^{k-2} \ll p n^{k-1}$.

Proof. Given $k, f, r$ and $c$ we choose $0<1 / n_{0} \ll \varepsilon \ll \gamma \ll 1 / f^{2}, c$. Fix $H, U$, and $y_{1}, \ldots, y_{r}$ satisfying the assumptions of the lemma. Without loss of generality we assume that $c p|U|^{k-1} \leq$ $\operatorname{deg}\left(y_{i} ; U\right) \leq(1+\varepsilon) c p|U|^{k-1}$ for each $i \in[r]$, by passing to a subgraph of $H$ if necessary. For a rooted linear $k$-graph $\left(F,\left(x_{1}, \ldots, x_{r}\right)\right)$ let $t_{F}=e(F)-\sum_{i \in[r]} \operatorname{deg}_{F}\left(x_{i}\right)$ denote the number of edges containing no root vertices. By induction on $t=t_{F}$ we show that for any such $\left(F,\left(x_{1}, \ldots, x_{r}\right)\right)$ on at most $r+f$ vertices and with edge degeneracy at most $\ell$, there are

$$
(1 \pm(t+1) \cdot \gamma) c^{e(F)-t} p^{e(F)}|U|^{v(F)-r}
$$

rooted copies of $\left(F,\left(x_{1}, \ldots, x_{r}\right)\right)$ in $\left(H,\left(y_{1}, \ldots, y_{r}\right), U\right)$. As $t_{F} \leq\left(\begin{array}{l}f \\ 2\end{array}\right)$ the lemma then follows by the choice of $\gamma$.

Consider first the case $t=0$, i.e., all edges of $F$ contain some root vertex. As $F$ is linear and edges of $F$ containing different root vertices are disjoint, a rooted copy of $F$ is simply a disjoint union of stars with each star centered at some $y_{i}$. The degree conditions therefore yield the correct count on the number of rooted copies. Indeed, for any $y_{i}$ and any set $X \subset U$ of at most $r+f$ vertices the number of edges containing $y_{i}$ and a vertex from $X$ is at most $|X| \Delta_{2}(H)$. Thus, for each $i \in[r]$ and each of the $\operatorname{deg}_{F}\left(x_{i}\right)$ edges in $F$ containing $x_{i}$ there are $\operatorname{deg}_{H}\left(y_{i} ; U\right) \pm(r+f) \Delta_{2}(H)=$ $(1 \pm \varepsilon) c p|U|^{k-1} \pm(r+f) \varepsilon p n^{k-1}$ ways to choose the image of this edge to build a copy of $F$. With $I$ denoting the number of isolated vertices of $F$, the number of rooted copies of $F$ in $H$ is then

$$
(1 \pm \gamma / 2) \prod_{i \in[r]}\left(c p|U|^{k-1}\right)^{\operatorname{deg}_{F}\left(x_{i}\right)}(|U| \pm(r+f))^{I}=(1 \pm \gamma)(c p)^{e(F)}|U|^{v(F)-r},
$$

proving the induction base.

For the induction step let $t \geq 1$, let $F$ be a $k$-graph with $t_{F}=t$, and let $\sigma$ be an edge exposure which certifies the edge degeneracy of $F$. Note that the induction hypothesis applies to any proper subgraph of $F$ as it has edge degeneracy at most $\ell$ and strictly fewer edges. Let $F^{\prime}=F_{t-1}=$ $\left(V(F), E(F) \backslash\left\{e_{q}\right\}\right)$ where $e_{q}$ is the last edge of $F$ according to the ordering $\sigma$, i.e., $q:=\sigma(t+$ $\left.\sum_{i=1}^{r} \operatorname{deg}_{F}\left(x_{i}\right)\right)$. Note that $F^{\prime}$ is defined on the same vertex set as $F$. For a labelled copy $T$ of $F^{\prime}$ in $H$ we denote by $K_{T}$ the $k$-set of vertices of $T$ which corresponds to $e_{q}$ in $F$. Let $\mathbf{1}_{H}:\left(\begin{array}{c}V \\ k\end{array}\right) \rightarrow\{0,1\}$ be the indicator function of the edge set of $H$. In this notation a copy $T$ of $F^{\prime}$ in $H$ extends to a copy of $F$ if and only if $\mathbf{1}_{H}\left(K_{T}\right)=1$, consequently, summing over all copies $T$ of $F^{\prime}$ in $H$ the number of copies of $F$ in $H$ is

$$
\sum_{T \subseteq H} \mathbf{1}_{H}\left(K_{T}\right)=\sum_{T \subseteq H}\left(p+\mathbf{1}_{H}\left(K_{T}\right)-p\right)=\sum_{T \subseteq H} p+\sum_{T \subseteq H}\left(\mathbf{1}_{H}\left(K_{T}\right)-p\right) .
$$

Noting that $e\left(F^{\prime}\right)=e(F)-1$ and $v\left(F^{\prime}\right)=v(F)$ the induction hypothesis yields

$$
\sum_{T \subseteq H} p=(1 \pm t \cdot \gamma) c^{e(F)-t} p^{e(F)}|U|^{v(F)-r}
$$

\footnotetext{
${ }^{5}$ Here and throughout, $\Delta_{2}(H)$ denotes the maximum 2-degree in $H$ i.e. $\Delta_{2}(H):=\max _{u \neq v \in V(H)} \mid\{e \in E(H)$ : $\{u, v\} \in e\} \mid$.
} 
and in the following we will give a bound to the error term $\sum_{T \subseteq H}\left(\mathbf{1}_{H}\left(K_{T}\right)-p\right)$.

Without loss of generality suppose that $e_{q}=\left\{u_{1}, \ldots, u_{k}\right\}$ and let $F_{*}=F\left[V(F) \backslash e_{q}\right]$ be the subhypergraph of $F$ obtained by removing the vertices $u_{1}, \ldots, u_{k}$. Due to linearity any edge in $E\left(F^{\prime}\right) \backslash E\left(F_{*}\right)$ intersects $e_{q}$ in at most one $u_{i}$. Hence, for any copy $T_{*}$ of $F_{*}$ there are sets $W_{i} \subseteq U$, $i \in[k]$, such that any $k$-tuple $K \in W_{1} \times \ldots \times W_{k}=$ : $\operatorname{ext}\left(T_{*}\right)$ extends $T_{*}$ to a copy of $F^{\prime}$. Explicitly, $W_{i}$ is the intersection of the neighbourhoods of the $(k-1)$-sets in $T_{*}$, which are the images of those $(k-1)$-sets in $F_{*}$ contained in an edge with $u_{i}$ in $F^{\prime}$. (Such a copy of $F^{\prime}$ then extends to a copy of $F$ if and only if $K \in E(H)$.) Let $z:=p^{w_{q}}|U|^{k}$, where $w_{q}$ is the weight of $e_{q}$ according to the edge exposure $\sigma$, that is $w_{q}=\sum_{i=1}^{k} \operatorname{deg}_{F^{\prime}}\left(u_{i}\right)$. Using that $w_{q} \leq \ell$ and Fact [1.8, we have that $H[U]$ is $\left(p, \varepsilon^{\prime} p^{w_{q}}, \varepsilon^{\prime}\right)$ pseudo-random for $\varepsilon^{\prime}=\sqrt{\varepsilon}$ (using that $\left.\varepsilon \ll c\right)$. There we obtain that

$$
\begin{aligned}
\left|\sum_{T \subseteq H}\left(\mathbf{1}_{H}\left(K_{T}\right)-p\right)\right| & \leq \sum_{T_{*} \subseteq H}\left|\sum_{K \in \operatorname{ext}\left(T_{*}\right)}\left(\mathbf{1}_{H}(K)-p\right)\right|=\sum_{T_{*} \subseteq H}\left|e\left(W_{1}, \ldots, W_{k}\right)-p\right| \operatorname{ext}\left(T_{*}\right)|| \\
& \leq \sum_{\substack{T_{*} \subseteq H \\
\left|\operatorname{ext}\left(T_{*}\right)\right| \geq \varepsilon^{\prime} z}} \varepsilon^{\prime} p\left|\operatorname{ext}\left(T_{*}\right)\right|+\sum_{\substack{T_{*} \subseteq H \\
\left|\operatorname{ext}\left(T_{*}\right)\right|<\varepsilon^{\prime} z}}\left(1+\varepsilon^{\prime}\right) \varepsilon^{\prime} p \cdot z .
\end{aligned}
$$

Here, the estimate for the first sum comes from the definition (1.3) whilst the second sum follows from Fact 1.8. Note that each edge in $E(F) \backslash\left(E\left(F_{*}\right) \cup\left\{e_{q}\right\}\right)$ contains exactly one vertex from $e_{q}$, hence we have $e(F)=e\left(F^{\prime}\right)+1=e\left(F_{*}\right)+w_{q}+1$. Thus, with $t_{*}=t_{F_{*}}<t$ denoting the number of root-free edges of $F_{*}=\left(F_{*},\left(x_{1}, \ldots, x_{r}\right)\right)$, we obtain from the induction hypothesis the following for the second sum in (2.2):

$$
\sum_{\substack{T_{*} \subseteq H \\ \operatorname{ext}\left(T_{*}\right) \mid<\varepsilon^{\prime} z}}\left(1+\varepsilon^{\prime}\right) \varepsilon^{\prime} p z \leq 2 c^{e\left(F_{*}\right)-t_{*}} p^{e\left(F_{*}\right)}|U|^{v(F)-k-r} \cdot\left(1+\varepsilon^{\prime}\right) \varepsilon^{\prime} p z \leq \frac{\gamma}{4} c^{e(F)-t} p^{e(F)}|U|^{v(F)-r} .
$$

To derive a bound for the first sum in (2.2), we will split the sum further. Define $J:=$ $\log 1 / \varepsilon^{\prime}+w_{q} \log 1 / p$ and for all $0 \leq j \leq J$, let $b_{j}$ be the number of copies $T_{*}$ of $F_{*}$ in $H$ such that $2^{j} \varepsilon^{\prime} z \leq\left|\operatorname{ext}\left(T_{*}\right)\right| \leq 2^{j+1} \varepsilon^{\prime} z$. Note that this covers all possible copies as $2^{J+1} \varepsilon^{\prime} z \geq|U|^{k}$. Then the number of rooted copies of $F^{\prime}$ in $H$ is at least $\sum_{j=0}^{J} b_{j} 2^{j} \varepsilon^{\prime} z$ and, by induction hypothesis, at most $2 p^{e\left(F^{\prime}\right)}|U|^{v\left(F^{\prime}\right)-r}=2 p^{e(F)-1}|U|^{v(F)-r}$. Consequently, the first sum in (2.2) is

$$
\begin{aligned}
\sum_{\substack{\left.T_{*} \subseteq H \\
\operatorname{ext}\left(T_{*}\right)|\leq| U\right|^{k}}} \varepsilon^{\prime} p\left|\operatorname{ext}\left(T_{*}\right)\right| & \leq \sum_{j=0}^{J} \sum_{\substack{T_{*} \subseteq H \\
2^{j} \varepsilon^{\prime} z \leq\left|\operatorname{ext}\left(T_{*}\right)\right| \leq 2^{j+1} \varepsilon^{\prime} z}} \varepsilon^{\prime} p\left|\operatorname{ext}\left(T_{*}\right)\right| \\
& \leq \sum_{j=0}^{J} b_{j} 2^{j+1} \varepsilon^{\prime 2} p z \leq 4 \varepsilon^{\prime} p^{e(F)}|U|^{v(F)-r} \leq \frac{\gamma}{4}(c p)^{e(F)}|U|^{v(F)-r} .
\end{aligned}
$$

Together with (2.2) and (2.3) we conclude that $\left|\sum_{T \subseteq H}\left(\mathbf{1}_{H}\left(K_{T}\right)-p\right)\right|<\frac{\gamma}{2} c^{e(F)-t} p^{e(F)}|U|^{v(F)-r}$ which finishes the proof of the lemma.

Remark 2.2. Note that if we take $r=0$ in Lemma 2.1, we can drop the maximum co-degree assumption as it is not required in the proof and so this gives Theorem 1.6. In fact, the proof of Lemma 2.1 actually establishes the stronger bounds (2.1), which under the same pseudo-randomness condition on $H$, yields the counting property for linear $k$-graphs. In general, the condition is tight 
up to a multiplicative constant as seen, e.g., by Alon's construction [5] of triangle-free $n$-vertex $d$ regular graphs with $d=\Omega\left(n^{2 / 3}\right)$, which are $(d / n, \lambda)$-jumbled with $\lambda=O\left(n^{1 / 3}\right)$. On the other hand the bound can be improved for other graphs, e.g., when $F$ is a larger odd cycle (see [39]).

2.2. Embedding compatible families. In this section we use the counting lemma, Lemma 2.1. to build a linear sized structure in the host $k$-graph. This structure is key to the absorption step but will also be useful in other parts of the proof. Let $(A, \mathcal{X})$ be a fixed rooted hypergraph with $r$ root vertices. The structure we look to find will consist of many rooted copies of $A$ in $H$, which respect a certain intersection restriction on the root vertices but are disjoint otherwise. Formally, let $T=\left(V_{T}, E\right)$ be a labelled $r$-graph with the vertex set $V_{T} \subset V(H)$ (which captures the intersection structure) 6 . Then $\left\{A_{e}\right\}_{e \in E(T)}$ is called a $T$-compatible family of copies of $(A, \mathcal{X})$ (or simply $T$ compatible) if:

(1) each $A_{e}, e \in E(T)$, is a rooted copy of $(A, \mathcal{X})$ in $(H, e, V(H))$, i.e., a rooted copy of $A$ in $H$ which maps $\mathcal{X}$ to $\mathcal{Y}=e$;

(2) each $A_{e}, e \in E(T)$, intersects $V_{T}$ exactly in $e$; and

(3) for any two edges $e, e^{\prime} \in E(T)$, the copies $A_{e}$ and $A_{e^{\prime}}$ intersect exactly in $e \cap e^{\prime}$.

In particular, note that the copies $A_{e}$ and $A_{e^{\prime}}, e \neq e^{\prime}$, are disjoint outside of $V(T)$. Our next lemma shows that if $T$ has bounded degree and our host hypergraph $H$ is suitably pseudo-random with respect to some $k$-graph $A$, then we can find some $T$-compatible family of copies of $A$.

Lemma 2.3. Given integers $k \geq 2, f, r \geq 0, \Delta \geq 1$ and $c>0$ there are $\varepsilon>0$ and $n_{0}$ such that for $n>n_{0}$ the following holds. Suppose that

- $(A, \mathcal{X})$ is a rooted linear $k$-graph with $r+f$ vertices, $r$ of which are roots,

- $H$ is a $\left(p, \varepsilon p^{\ell}, \varepsilon\right)$-pseudo-random n-vertex $k$-graph, with $\ell \geq \operatorname{degen}(A, \mathcal{X})$, and $\Delta_{2}(H)<$ $\varepsilon p n^{k-1}, Y \subset V(H)$ with $|Y| \leq \frac{n}{200 \Delta^{2}(r+f)^{2}}$ and $\operatorname{deg}_{H}(v ; V \backslash Y)>c p n^{k-1}$ for all $v \in Y$,

- $T$ is an ordered $r$-graph on the vertex set $V_{T}=Y$ with maximum vertex degree $\Delta_{1}(T) \leq \Delta$.

Then there exists a T-compatible family of rooted copies of $(A, \mathcal{X})$ in $H$.

We note here again that by Lemma 1.9 the condition $\Delta_{2}(H)<\varepsilon p n^{k-1}$ can be dropped if $\ell \geq k-1$.

Proof. Let integers $k, f, r, \Delta$ and $c$ be given. We choose $\gamma=\frac{1}{100 \Delta f}$ and as $c>0$ only appears in the lower bound for the degree condition we may assume that $c<\gamma / 8$. We apply the counting lemma, Lemma 2.1, with the parameters $k, f, r, q 2.1=\frac{c}{2^{k}}$ to obtain 92.1 . We choose $\varepsilon=\varepsilon 2.1 \frac{(c \gamma)^{k}}{4^{k} f \Delta}$ and let $n_{0}$ be sufficiently large. Let $(A, \mathcal{X}), H$ and $T$ with $V_{T}=Y$ be as in the lemma.

The idea is to construct the required family of rooted copies of $(A, \mathcal{X})$ by repeatedly using the greedy type Algorithm 1, which simply extends the family of rooted hypergraphs when it can and records the failure otherwise.

This greedy approach almost succeeds in finding all the copies of $(A, \mathcal{X})$ but we can not guarantee that it will $\log$ no failures. To deal with the small number of failures, we have to run the algorithm several times. First, we analyse the following simple case where we can successfully embed a small number of copies.

Claim 2.4. Suppose that $\hat{T} \subset T$ is a subhypergraph of $T$ with $e(\hat{T}) \leq \frac{c^{k}}{4 f}$ pn and $X \subset V(H) \backslash V_{T}$ is a set of size $|X| \geq c$ so that $\operatorname{deg}(v ; X)>c p|X|^{k-1}$ holds for each $v$ contained in an edge of $\hat{T}$. Then there is a $\hat{T}$-compatible family of rooted copies of $(A, \mathcal{X})$ whose vertices are entirely contained in $V_{T} \cup X$.

Proof of Claim 2.4. We run the Algorithm 1 with input $(A, \mathcal{X}), H, \hat{T}$ and $X$ and claim that the family $\mathcal{A}^{t}, t=e(\hat{T})$, produced by the algorithm, has the required properties. Let $\left(e_{1}, \ldots, e_{t}\right)$ be

\footnotetext{
${ }^{6}$ Note that each edge $e \in E=E(T)$ is a labelled $r$-set in $V_{T} \subset V(H)$.
} 


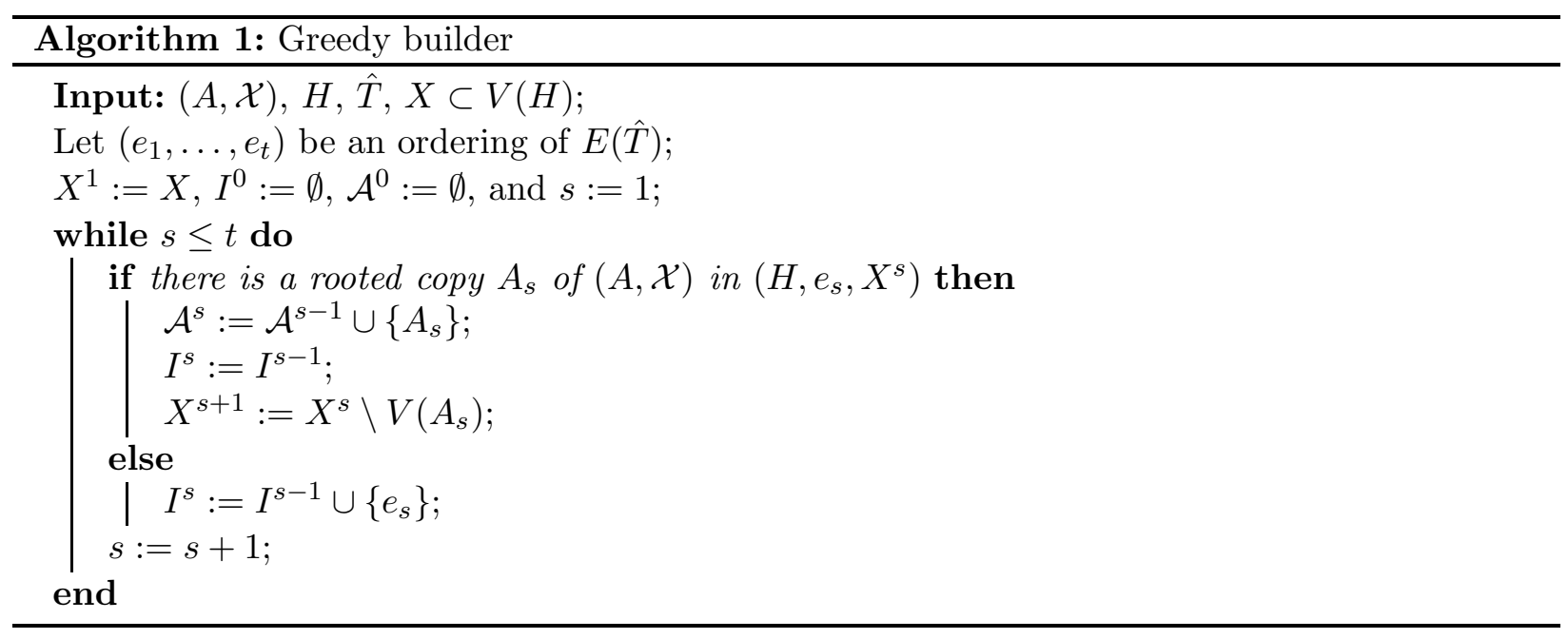

an ordering of $E(\hat{T})$. Note that after step $s \in[t]$ the algorithm has removed from $X$ in total at most $f \cdot s$ vertices. Thus, at each time $s$ the set $X^{s}$ in the algorithm has size $\left|X^{s}\right| \geq \frac{1}{2}|X|>$ $q_{2.1}^{n}$ and $\operatorname{deg}\left(v ; X^{s}\right) \geq \operatorname{deg}(v ; X)-f s \cdot \Delta_{2}(H)>q_{2.1}^{p}\left|X^{s}\right|^{k-1}$ holds for each $v \in e_{s}$, using that $\Delta_{2}(H) \leq n^{k-2}$. Thus, Lemma 2.1 applied with the choices of constants yields a rooted copy $A_{s}$ of $(A, \mathcal{X})$ in $\left(H, e_{s}, X^{s}\right)$. As this holds for all $s \in[t]$ the family $\mathcal{A}^{t}$ is $\hat{T}$-compatible and is contained in $V_{T} \cup X$.

Returning to the proof of Lemma 2.3, choose disjoint subsets $U, W \subset V(H) \backslash Y$ of size $|U|=$ $|W|=2 \gamma n$ but arbitrarily otherwise. Let

$$
B=\left\{v \in V_{T}: \operatorname{deg}(v ; W)<2 c p|W|^{k-1}\right\}
$$

and let $T_{1} \subset T$ denote the subgraph of $T$ on the same vertex set $V_{T}$ which consists of all edges intersecting $B$. By the pseudo-randomness of $H$ we conclude that $|B| \leq \varepsilon \gamma^{-(k-1)} p^{\ell} n$. As $e\left(T_{1}\right) \leq$ $\Delta|B|$ we can apply Claim 2.4 with $\hat{T}=T_{1}$ and $X=V(H) \backslash V_{T}$ to find a $T_{1}$-compatible family $\mathcal{A}_{1}$. Let $U^{\prime}=U \backslash V\left(\mathcal{A}_{1}\right)$ and $W^{\prime}=W \backslash V\left(\mathcal{A}_{1}\right)$ which are disjoint sets of size $\left|U^{\prime}\right|,\left|W^{\prime}\right| \geq \gamma n$ and note that each vertex $v \in V_{T} \backslash B$ satisfies

$$
\operatorname{deg}\left(v ; W^{\prime}\right) \geq \operatorname{deg}(v ; W)-f \cdot e\left(T_{1}\right) \Delta_{2}(H)>c p\left|W^{\prime}\right|^{k-1} .
$$

Claim 2.5. Let $T^{\prime} \subset T$ be the subgraph obtained by removing the edge set $E\left(T_{1}\right)$ from $T$. Then there is a subgraph $T_{2} \subset T^{\prime}$ with $e\left(T_{2}\right) \geq e\left(T^{\prime}\right)-(2 / \gamma)^{k-1} \Delta \varepsilon p^{\ell} n$ and a $T_{2}$-compatible family $\mathcal{A}_{2}$ of rooted copies of $(A, \mathcal{X})$ whose vertices are entirely contained in $V_{T} \cup U^{\prime}$.

Before proving the claim we note that it readily implies the lemma. Indeed, define $T_{3}=T^{\prime} \backslash T_{2}$ which then satisfies $e\left(T_{3}\right) \leq(2 / \gamma)^{k-1} \Delta \varepsilon p^{\ell} n$. Further, (2.4) holds for all vertices in $V_{T} \backslash B$, in particular for all those contained in edges of $T_{3}$. Thus we can apply Claim 2.4 with $\hat{T}=T_{3}$ and $X=W^{\prime}$ and obtain a $T_{3}$-compatible family $\mathcal{A}_{3}$ of rooted copies of $(A, \mathcal{X})$ whose vertices are entirely contained in $V_{T} \cup W^{\prime}$. Since $T=T_{1} \cup T_{2} \cup T_{3}$, the family $\mathcal{A}_{1} \cup \mathcal{A}_{2} \cup \mathcal{A}_{3}$ is $T$-compatible and the lemma follows.

Proof of Claim [2.5. We run the Algorithm 1 with input $(A, \mathcal{X}), H, \hat{T}=T^{\prime}$ and $X=U^{\prime}$ and it is sufficient to show that $\left|I^{t}\right| \leq(2 / \gamma)^{k-1} \Delta \varepsilon p^{\ell} n$. Let $\left(e_{1}, \ldots, e_{t}\right)$ be an ordering of $E\left(T^{\prime}\right)$. As $t<\Delta\left|V_{T}\right| \leq \frac{\gamma n}{2 f} \leq \frac{|X|}{2 f}$ and at each time $s<t$ the algorithm removes at most $f$ vertices from $X^{s}$

\footnotetext{
${ }^{7}$ Here, $V(\mathcal{A})$ denotes the set of vertices which feature in rooted copies of $A$ in the family $\mathcal{A}$.
} 
to obtain $X^{s+1}$, we have for each time $s \in[t]$ that $\left|X^{s}\right| \geq\left|X^{1}\right|-(s-1) f>\left|X^{1}\right| / 2 \geq \gamma n / 2$. Then Lemma 2.1 applied with the choices of constants implies that for each $s \in[t]$ there is a rooted copy of $(A, \mathcal{X})$ in $\left(H, e_{s}, X^{s}\right)$ unless the degree condition fails for a vertex in $e_{s}$. Thus, $I^{t}$ comprises exactly of those $e_{s}$ such that for some vertex in $e_{s}$, say $y^{s}$, we have $\operatorname{deg}\left(y^{s} ; X^{s}\right)<$ 2.1. $^{p}\left|X^{s}\right|^{k-1} \leq q_{2.1}|X|^{k-1}$, which implies that $\operatorname{deg}\left(y^{s} ; X^{t}\right)<q 2.1 p|X|^{k-1} \leq c p\left|X^{t}\right|^{k-1}$. Let

$$
Y^{t}=\left\{y \in V_{T}: \operatorname{deg}\left(y ; X^{t}\right)<c p\left|X^{t}\right|^{k-1}\right\} .
$$

Clearly, $e\left(Y^{t}, X^{t}, \ldots, X^{t}\right) \leq c p\left|Y^{t}\right|\left|X^{t}\right|^{k-1}$ and the pseudo-randomness condition together with $\left|X^{t}\right| \geq \gamma n / 2$ implies that $\left|Y^{t}\right| \leq(2 / \gamma)^{k-1} \varepsilon p^{\ell} n$. On the other hand, for any $e_{s} \in I^{t}$, the vertex $y^{s} \in e_{s}$, as detailed above, is contained in $Y^{t}$ and every $y \in Y^{t}$ is $y^{s}$ for at most $\Delta$ elements $e_{s} \in I$. This shows that $\left|I^{t}\right| \leq \Delta\left|Y^{t}\right|$ and the claim follows.

\section{Absorbers And the template}

As mentioned in Section 1.1 our proof works by absorption. In particular, it utilises the "absorber-template" method introduced by Montgomery [46, 47] which has since been used by various authors in different settings, see, e.g., [20, 21, 28, 30, 41, 49. In our case we combine many copies of small special subgraphs called absorbers to a large family using a large template, which captures how these copies intersect. The absorbers will depend on the spanning structure we are interested in but in both cases we should be able to find them in the pseudo-random host, thus they need to be linear hypergraphs and edges containing distinct rooted vertices should be disjoint. Moreover, in light of Lemma 2.1 we want that absorbers have small edge degeneracy as to weaken the pseudo-random condition necessary for the argument. The absorbers for factors given here are straightforward to describe and rely on permuting copies of $F$ in a grid-like structure so as to reduce the degeneracy. The path absorbers, however, are more involved and differ from those used in absorbing arguments before. In particular, the first absorbers [9,27] for finding loose Hamilton cycles in hypergraphs do not satisfy our definition of path absorbers, while the ones used, e.g., in [45], have edge degeneracy $k$ and so are not as effective as those given here, which have degeneracy $k-1$.

3.1. Absorbers for factors. For a linear $k$-graph $F$ an absorber for $F$ is a rooted linear $k$-graph $\left(A_{F}, \mathcal{X}\right)$ with non-empty root vector $\mathcal{X}$ such that there is an $F$-factor of $A_{F}$ and an $F$-factor of the subgraph of $A_{F}$ obtained by removing all roots $\mathcal{X}$. We will often refer to the $F$-factor on the full vertex set of $A_{F}$ as the complete $F$-factor, while the factor on $V\left(A_{F}\right) \backslash \mathcal{X}$ is referred to as the internal $F$-factor. Note that the number of root vertices is a multiple of $|V(F)|$.

Lemma 3.1. Given $k$ and a linear $k$-graph $F$ on $[f]$ vertices. Then there is an absorber $\left(A_{F}, \mathcal{X}\right)$ of $F$ with $f^{2}$ vertices, $f$ roots and edge degeneracy

$$
\operatorname{degen}\left(A_{F}, \mathcal{X}\right) \leq \operatorname{degen}(F)+\max _{e \in E(F)} \sum_{u \in e} \operatorname{deg}_{F}(u)=\operatorname{degen}(F)+\Delta^{\prime}(F)+k,
$$

where degen $(F)$ is considered here to be the degeneracy of $F$ with an empty root set of vertices. In particular, if $F$ consists of a single edge, then there is an absorber of edge degeneracy at most $k$.

Proof. Given a linear $k$-graph $F$ on the vertex set $V(F)=\mathbb{Z}_{f}=\mathbb{Z} / f \mathbb{Z}$ we define the $k$-graph $A_{F}$ on the vertex set $\mathbb{Z}_{f} \times \mathbb{Z}_{f}$ as follows. For each $i \in \mathbb{Z}_{f}, A_{F}$ contains a copy $F_{i}$ of $F$ on the vertex set $V\left(F_{i}\right)=\{i\} \times \mathbb{Z}_{f}$. Further, for each $j \in[f-1], A_{F}$ contains a copy $F^{j}$ of $F$ on the vertex set $V\left(F^{j}\right)=\left\{(i, i+j): i \in \mathbb{Z}_{f}\right\}$ where addition is in $\mathbb{Z}_{f}$. These copies we place so that the projection to the second coordinate $\varphi((\cdot, \ell))=\ell$ defines an isomorphism between $F_{i}$ and $F$ and between $F^{j}$ and $F$, respectively. Note that $A_{F}$ is a linear hypergraph since $F_{i}$ and $F_{j}$ are disjoint for $i j \in \mathbb{Z}_{f} \times \mathbb{Z}_{f}$, $i \neq j$, and $F^{i}$ and $F^{j}$ are disjoint for $i j \in[f-1] \times[f-1], i \neq j$, while $F_{i}$ and $F^{j}, i j \in \mathbb{Z}_{f} \times[f-1]$ intersect only in the vertex $(i, i+j)$. We further define roots $\mathcal{X}=((1,1), \ldots,(f, f))$ and obtain the 
rooted $k$-graph $\left(A_{F}, \mathcal{X}\right)$ with the property that $\left\{F_{i}\right\}_{i \in \mathbb{Z}_{f}}$ gives the complete $F$-factor of $A_{F}$ while $\left\{F^{j}\right\}_{j \in[f-1]}$ gives the internal $F$-factor. Hence, $A_{F}$ is an absorber of $F$ and it remains to show the bound on the degeneracy of $A_{F}$.

Let $\sigma$ denote an edge exposure of $F$ (without any roots) which yields the degeneracy of $F$. We construct an edge exposure $\tau$ for $A_{F}$ by first exposing edges containing the roots in an arbitrary order. Note that this step does not expose any edge of $F^{j}, j \in[f-1]$, since none of them contains root vertices. In the second step we expose the remaining edges of all $F_{i}, i \in \mathbb{Z}_{f}$ in an arbitrary order and finally, in the third step, we expose the edges of each $F^{j}, j=1, \ldots, f-1$ according to the order given by $\sigma$. As the $F_{i}$ 's are vertex disjoint each edge $e$ from the second step has weight at most $\sum_{u \in e}\left(\operatorname{deg}_{F}(u)-1\right)$.

To bound the weights of the edges in the third step consider a $j \in[f-1]$ and let $e_{t}=\left\{\left(i_{1}, i_{1}+\right.\right.$ $\left.j), \ldots,\left(i_{k}, i_{k}+j\right)\right\}$ be the $t$-th edge of $F^{j}$ in the ordering $\tau$. Recall that $F^{j}$ is disjoint from other $F^{j^{\prime}}$, that we expose the edges of $F^{j}$ according to $\sigma$ and that $V\left(F_{i}\right) \cap V\left(F^{j}\right)=\{(i, i+j)\}$ for $i \in \mathbb{Z}_{f}$. Therefore, the weight of $e_{t}$ with respect to $\tau$ is exactly

$$
w_{\sigma(t)}+\sum_{\ell \in[k]} \operatorname{deg}_{F_{i_{\ell}}}\left(\left(i_{\ell}, i_{\ell}+j\right)\right) .
$$

Recall also that $F_{i}$ and $F^{j}$ are identical copies of $F$, i.e., the projection to the second coordinate $\varphi(\cdot, \ell)=\ell$ is an isomorphism of $F_{i}$ and $F^{j}$ to $F$. By this projection $\left\{i_{1}+j, \ldots, i_{k}+j\right\}$ is an edge in $F$ and the degree of $\left(i_{\ell}, i_{\ell}+j\right)$ in $F_{i_{\ell}}$ is $\operatorname{deg}_{F}\left(i_{\ell}+j\right)$ for each $\ell \in[k]$. Hence, $\sum_{\ell \in[k]} \operatorname{deg}_{F_{i_{\ell}}}\left(\left(i_{\ell}, i_{\ell}+j\right)\right) \leq$ $\max _{e \in E(F)} \sum_{u \in e} \operatorname{deg}_{F}(u)$. Together with $w_{\sigma(t)} \leq \operatorname{degen}(F)$ this yields the desired bound.

3.2. Path absorbers. A $k$-uniform path absorber is a rooted linear $k$-graph $\left(P, \mathcal{X}, y_{1}, y_{2}\right)$ with a non-empty set of root vertices $\mathcal{X}$ and two distinguished vertices $y_{1}, y_{2} \in V(P) \backslash \mathcal{X}$, called end vertices, such that there is a loose path from $y_{1}$ to $y_{2}$ which uses all the vertices of $V(P)$ and a loose path from $y_{1}$ to $y_{2}$ in $P$ which covers the vertices $V(P) \backslash \mathcal{X}$. The first path we call the complete loose path and latter is called the internal loose path.

Lemma 3.2. For each $k \geq 3$, there exists a $k$-uniform path absorber $\left(P, \mathcal{X}, y_{1}, y_{2}\right)$ on $9 k^{2}-23 k+15$ vertices with roots $\mathcal{X}=\left\{x_{1}, \ldots, x_{k-1}\right\}$ and degeneracy $\operatorname{degen}(P, \mathcal{X})=k-1$.

Proof. Our path absorber is defined by smaller subgraphs which we call absorbing gadgets (see Figure 11). An absorbing gadget $P_{i}$ is a hypergraph on $5 k-6$ vertices, with disjoint vertex subsets $A_{i}$, $A_{i}^{\prime}, B_{i}$ and $C_{i}$ such that $\left|A_{i}\right|=\left|A_{i}^{\prime}\right|=k-2,\left|B_{i}\right|=k-3,\left|C_{i}\right|=2 k+1$ and $V\left(P_{i}\right)=A_{i} \cup A_{i}^{\prime} \cup B_{i} \cup C_{i}$. We label $C_{i}=\left\{c_{i 1}, \ldots, c_{i k}, c_{i 1}^{\prime}, \ldots, c_{i k}^{\prime}, c_{i *}\right\}$ and let $E\left(P_{i}\right)=\left\{e_{i}, e_{i}^{\prime}, f_{i}, f_{i}^{\prime}, g_{i}\right\}$ where

$$
\begin{aligned}
e_{i} & :=\left\{c_{i 2}, c_{i *}\right\} \cup A_{i}, \quad f_{i}:=\left\{c_{i 1}, \ldots, c_{i k}\right\}, \\
e_{i}^{\prime} & :=\left\{c_{i 2}^{\prime}, c_{i *}\right\} \cup A_{i}^{\prime}, \quad f_{i}^{\prime}:=\left\{c_{i 1}^{\prime}, \ldots, c_{i k}^{\prime}\right\} \quad \text { and } \\
g_{i}:=\left\{c_{i k}, c_{i *}, c_{i 1}^{\prime}\right\} \cup B_{i} . &
\end{aligned}
$$

The key property of the absorbing gadget is that there are two loose paths in $P_{i}$, both with end vertices $c_{i 1}$ and $c_{i k}^{\prime}$. Indeed, there is a loose path from $c_{i 1}$ to $c_{i k}^{\prime}$ which covers $V(P) \backslash\left(A_{i} \cup A_{i}^{\prime}\right)$, namely taking the edge sequence $\left(f_{i}, g_{i}, f_{i}^{\prime}\right)$. We call this the inner loose path of $P_{i}$. We also have the outer loose path of $P_{i}$ defined by the edge sequence $\left(f_{i}, e_{i}, e_{i}^{\prime}, f_{i}^{\prime}\right)$ which covers $V(P) \backslash B_{i}$ and also has endpoints $c_{i 1}$ and $c_{i k}^{\prime}$.

The path absorber $P$ is then defined by taking copies of these absorbing gadgets, joining them together using singular edges and identifying vertices in $A_{i} \cup A_{i}^{\prime} \cup B_{i}$ across the gadgets. In more detail, we take the vertex set of $P$ to be the disjoint union

$$
V(P):=\mathcal{X} \cup U \cup V \cup W \cup \bigcup_{i=1}^{2 k-3} C_{i} \cup \bigcup_{i=1}^{2 k-4} D_{i} .
$$




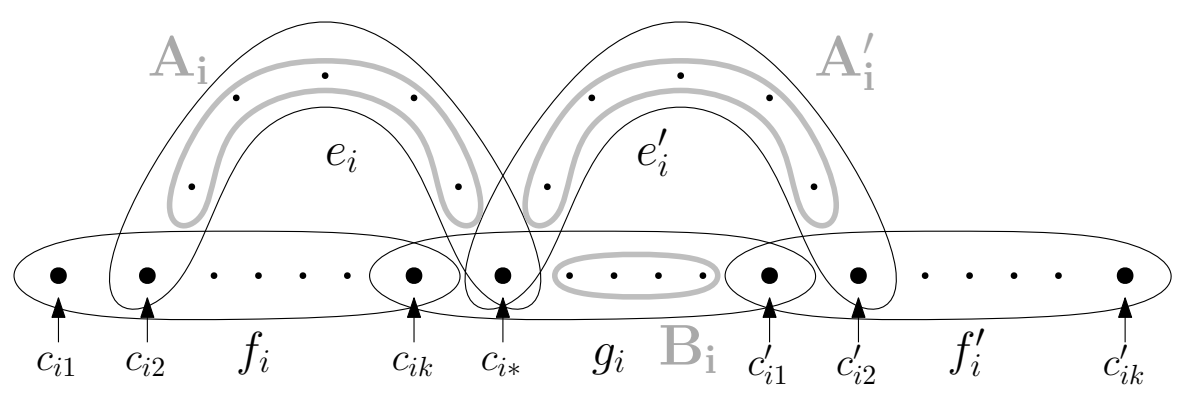

FiguRE 1. An absorbing gadget.

On $V(P)$ we will define absorbing gadgets $P_{1}, \ldots, P_{2 k-3}$, where $V\left(P_{i}\right)=A_{i} \cup A_{i}^{\prime} \cup B_{i} \cup C_{i}$ and $E\left(P_{i}\right)=\left\{e_{i}, e_{i}^{\prime}, f_{i}, f_{i}^{\prime}, g_{i}\right\}$ as above. The set $C_{i}$ is also labelled as in the previous paragraph and is used by $P_{i}$ only, while vertices in $A_{i}, A_{i}^{\prime}$ and $B_{i}$ will be shared with other gadgets in the way explained in the next paragraph. The sets $D_{i},\left|D_{i}\right|=k-2$, are used to build the edges $h_{i}=\left\{c_{i k}^{\prime}, c_{(i+1) 1}\right\} \cup D_{i}$, $i \in[2 k-4]$ which connect $P_{i}$ and $P_{i+1}$. All together we obtain edge set of $P$ given by

$$
E(P):=E\left(P_{1}\right) \cup \cdots \cup E\left(P_{2 k-3}\right) \cup\left\{h_{1}, \ldots, h_{2 k-4}\right\}
$$

and we take $y_{1}:=c_{11}$ and $y_{2}:=c_{(2 k-3) k}^{\prime}$ to be the two endpoints of $P$.

To complete the definition of $P$, it is left to assign the vertices in $A_{i}, A_{i}^{\prime}$ and $B_{i}$ as subsets of $\mathcal{X} \cup U \cup V \cup W$. For this purpose we consider the following labelling

$$
\begin{aligned}
\mathcal{X} & :=\left\{x_{1}, \ldots, x_{k-1}\right\}, \\
U & :=\left\{u_{i j}: 1 \leq i, j \leq k-1 \text { and } i \bmod (k-1) \notin\{j, j-1\}\right\}, \\
W & :=\left\{w_{i j}: k \leq i, j \leq 2 k-3 \text { and } i \neq j\right\}, \\
V & :=\left\{v_{i j}: 1 \leq i \leq k-1 \text { and } k \leq j \leq 2 k-3\right\} .
\end{aligned}
$$

We assign the vertices as follows. For $1 \leq i \leq k-1$ we define

$$
\begin{aligned}
& A_{i}:=\left\{x_{i}\right\} \cup\left\{u_{i \ell}: 1 \leq \ell \leq k-1 \text { and } \ell \bmod (k-1) \notin\{i, i+1\}\right\}, \\
& A_{i}^{\prime}:=\left\{v_{i \ell}: k \leq \ell \leq 2 k-3\right\}, \\
& B_{i}:=\left\{u_{\ell i}: 1 \leq \ell \leq k-1 \text { and } \ell \bmod (k-1) \notin\{i-1, i\}\right\} .
\end{aligned}
$$

On the other hand, for $k \leq i \leq 2 k-3$, we assign the vertices in the following way:

$$
\begin{aligned}
& A_{i}:=\left\{v_{(i-k+1) i}\right\} \cup\left\{w_{i \ell}: k \leq \ell \leq 2 k-3 \text { and } \ell \neq i\right\}, \\
& \left.A_{i}^{\prime}:=\left\{v_{\ell i}: 1 \leq \ell \leq k-1 \text { and } \ell \neq i-k+1\right)\right\} \quad \text { and } \\
& B_{i}:=\left\{w_{\ell i}: k \leq \ell \leq 2 k-3 \text { and } \ell \neq i\right\} .
\end{aligned}
$$

This finishes the definition of $P$. Examples of these vertex set assignments are shown in Figure 2 , When $k=3$, the sets $B_{i}$ are empty which simplifies the situation considerably. The resulting hypergraph in this case is shown in Figure 3 .

Let us now establish the claimed properties. Firstly, it is easily verified that $V(P)$ has the required size and we now show that $P$ is linear. Note that for a fixed $i \in[2 k-3]$, the vertices that appear in the absorbing gadget $P_{i}$ are all distinct. One can easily check that $P_{i}$ is linear and the edges $h_{i}$ intersect two distinct gadgets in one vertex each. Therefore it suffices to establish that if $e \in E\left(P_{i}\right)$ and $f \in E\left(P_{j}\right)$, for $i \neq j$ then $|e \cap f| \leq 1$. If $i, j \in[k-1], i \notin\{j-1, j+1\} \bmod (k-1)$, then $V\left(P_{i}\right) \cap V\left(P_{j}\right)=\left\{u_{i j}, u_{j i}\right\}$ and these vertices do not lie in the same edge, neither in $P_{i}$ nor in $P_{j}$. If $i \in\{j-1, j+1\} \bmod (k-1)$, then the situation is even simpler as $\left|V\left(P_{i}\right) \cap V\left(P_{j}\right)\right| \leq 1$. In a similar fashion, we have that if $k \leq i, j \leq 2 k-3$ then $V\left(P_{i}\right) \cap V\left(P_{j}\right)=\left\{w_{i j}, w_{j i}\right\}$ and neither $P_{i}$ nor $P_{j}$ 


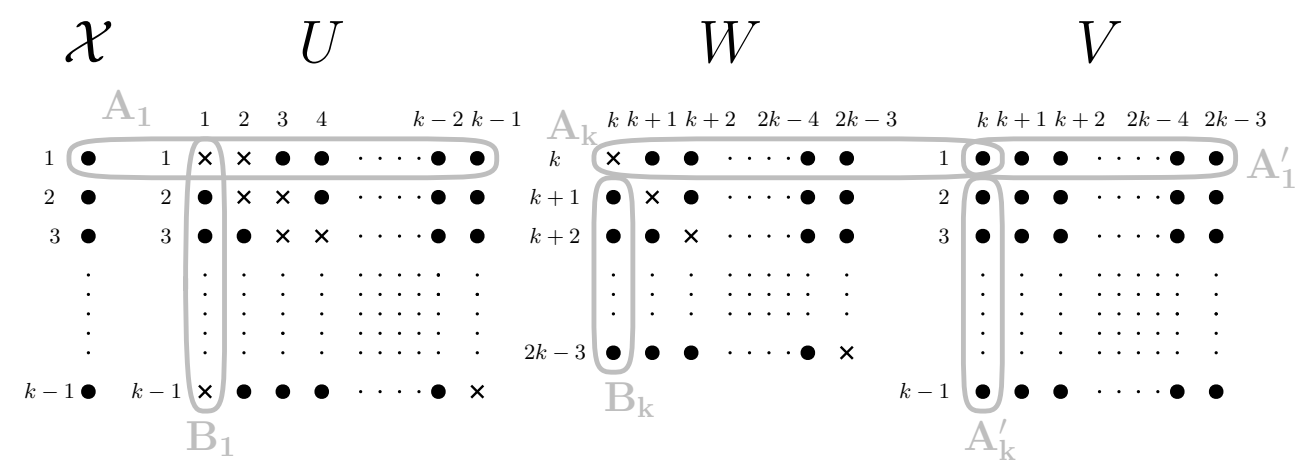

Figure 2. Assigning the vertices.

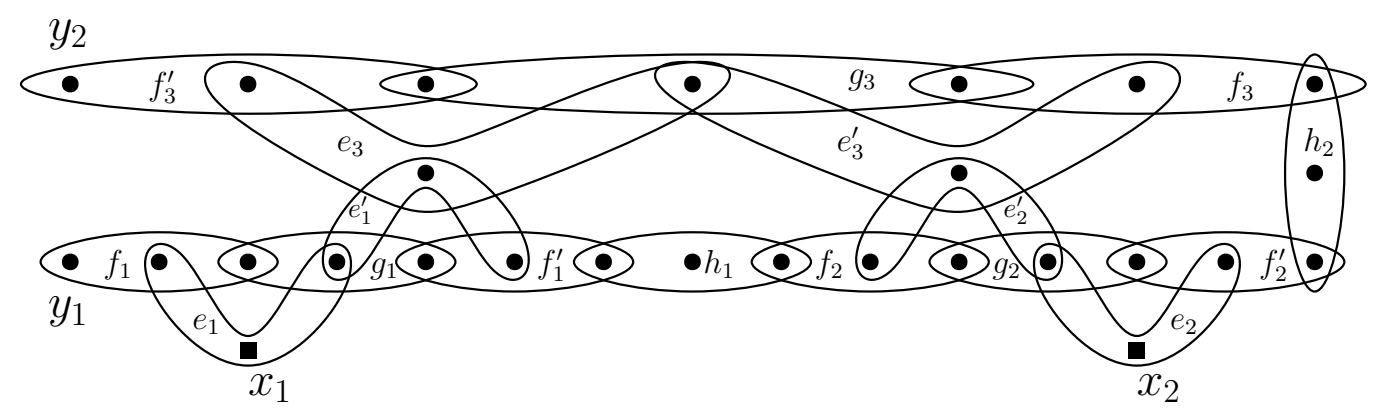

FiguRE 3. A three-uniform path absorber.

have an edge which contains both of these vertices. Finally if $1 \leq i \leq k-1$ and $k \leq j \leq 2 k-3$, then $V\left(P_{i}\right) \cap V\left(P_{j}\right)=\left\{v_{i j}\right\}$.

We now verify that $\left(P, \mathcal{X}, y_{1}, y_{2}\right)$ defines a path absorber. Consider traversing the outer loose path of $P_{i}$ for $1 \leq i \leq k-1$ and the inner loose path for $k \leq i \leq 2 k-3$ as well as the edges $h_{i}$ which link the absorbing gadgets. This gives a loose path from $y_{1}$ to $y_{2}$, which covers all the vertices exactly once. Indeed, when we traverse the outer loose paths in the first $k-1$ absorbing gadgets we cover all of $U, V$ and $\mathcal{X}$ exactly once whilst avoiding the $B_{i}$ and thus avoiding taking any $u \in U$ more than once. Further, taking the inner loose paths for $k \leq i \leq 2 k-3$ guarantees that we cover $W$ and do not repeat any vertices of $V$ in the process. Similarly, if we now consider taking the inner loose path for all the absorbing gadgets $P_{i}$ with $1 \leq i \leq k-1$ and the outer loose path for all absorbing gadgets $P_{i}$ with $k \leq i \leq 2 k-3$, one can see that we obtain a loose path from $y_{1}$ to $y_{2}$ on $P$ which covers exactly the vertices $V(P) \backslash \mathcal{X}$.

Finally let us turn to the degeneracy of $(P, \mathcal{X})$. We consider the following edge exposure. We reveal all the $e_{i}$ for $i=1,2, \ldots, 2 k-3$ first. This guarantees that all the edges with root vertices are revealed first. We then reveal $e_{i}^{\prime}$ for $i=1,2, \ldots, 2 k-3$. Each $e_{i}^{\prime}$ has weight at most $k-1$. Indeed, as we add $e_{i}^{\prime}$ the vertex $c_{i 2}^{\prime}$ contributes nothing to the weight, whilst all other vertices contribute at most one. We then reveal $g_{i}$ for each $i=1,2, \ldots, 2 k-3$. Again we can conclude that the weight of $g_{i}$ according to this edge exposure is at most $k-1$ as no edges containing $c_{i k}$ or $c_{i 1}^{\prime}$ have been revealed yet and so they contribute nothing to the weight, whilst $c_{i *}$ contributes two and all other vertices contribute at most one. Indeed all other vertices in the edge have degree two and so can contribute no more than one to the weight. We can then reveal $f_{i}$ and $f_{i}^{\prime}$ for $i=1,2, \ldots, 2 k-3$, each of which has weight two, given by the two vertices in the edge which have previously featured. Finally, we reveal $h_{i}$ for $i=1,2, \ldots, 2 k-4$ which again has weight two given by its two degree two vertices. This gives an edge exposure with degeneracy $k-1$. 
3.3. The template. We look to find many (path) absorbers in our host graph and the relative positions of these absorbers will be determined by an auxiliary hypergraph which we call a template.

Definition 3.3. An $(r, m)$-template $T$ is an $r$-uniform, $r$-partite hypergraph with vertex parts $V(T)=Y_{0} \cup Y_{1} \cup \ldots \cup Y_{r-1}$ of sizes $\left|Y_{0}\right|=4 m,\left|Y_{1}\right|=\cdots=\left|Y_{r-1}\right|=3 m$ such that the following holds. There exists a subset $Z \subset Y_{0}$, called the flexible set, of size $|Z|=2 m$ with the property that for any $Z^{\prime} \subset Z$ of size $m$ the induced hypergraph $T\left[V \backslash Z^{\prime}\right]$ has a perfect matching.

As $T$ is $r$-uniform and $r$-partite there is no confusion in considering edges of $T$ as sets or as ordered tuples and we will switch between these viewpoints throughout. For large enough $m$ the existence of a $(2, m)$-template with maximum vertex-degree $\Delta_{1}(T) \leq 40$ was proven by Montgomery [46, 47] using a probabilistic argument. These easily extend to $(r, m)$-templates by taking $r-2$ disjoint copies $Y_{2}, \ldots, Y_{r-1}$ of $Y_{1}$ and adding to each edge $a b \in\left(Y_{0} \times Y_{1}\right) \cap E(T)$ the copies $b^{i} \in Y_{i}$ of $b \in Y_{1}$, $i \in\{2, \ldots, r-1\}$ to make it $r$-uniform. This yields the following.

Lemma 3.4. For an $r \geq 2$ and large enough $m$ there is an $(r, m)$-template $T$ with $\Delta_{1}(T) \leq 40$.

Templates $T$ are defined so that they are flexible with respect to perfect matchings in $T$, reflected by the existence of the flexible set $Z \subset V(T)$. Let $T$ with $V(T) \subset V(H)$ be a suitable chosen template. By combining this with the notions of absorbers from Section 3.1] and Section [3.2, and that of $T$-compatible families from Section 2.2, one can transform $V(T)$ into a set $A \supset V(T)$ such that $Z \subset A$ is flexible with respect to the desired spanning structure, which means that for each $Z^{\prime} \subset Z$ of size $m$ the induced $k$-graph $H\left[A \backslash Z^{\prime}\right]$ contains an $F$-factor or a spanning loose path with end vertices independent of $Z^{\prime}$. This is the key property in our proof of the main theorems.

\section{Proof of Theorem 1.7 And Theorem 1.5}

We prove both theorems, Theorem 1.7 and Theorem 1.5, at once following the outline from Section 1.1. The proof essentially consists of two steps, encapsulated by Claim 4.2 and Claim 4.3 . The first, Claim 4.2, concerns the construction of the flexible and absorbing sets $Z \subset A \subset V(H)$ as explained in the outline given in Section 1.1 and in the last paragraph of the previous section. The second, Claim 4.3, then makes use of the flexibility of $Z$ to construct a spanning structure in $H\left[(V \backslash A) \cup Z^{\prime}\right]$ for some suitable $Z^{\prime} \subset Z$. Combining this with the spanning structure in $H\left[A \backslash Z^{\prime}\right]$, which exists by the flexibility of $Z$, we obtain the desired spanning structure of $H$.

Before delving into the proof let us first expand the outline of the construction of the absorbing set from Claim 4.2 by some technical details and explain the role that Claim 4.1 plays. To be able to make use of the flexible set $Z \subset V(H)$ we require that essentially all vertices have high degree into $Z$, including those in $Z$. Due to the necessity to connect paths we require slightly more in the Hamilton cycle case, namely that these vertices have high degree, e.g., into sets $Z_{1}$ and $Z_{2}$ of a partition of $Z$ and to another set $W$, which we use within the claim to connect the many small path absorbers to one long path. One way to ensure this degree condition is to simply choose a slightly larger set and remove vertices with bad degree, of which there are few due to the pseudo-randomness. Using Claim 4.1 these vertices can then be covered by a small $F$-factor or a loose path, respectively, giving rise to the additional set $U$ in Claim 4.2, Claim 4.1 will also become handy in the proof of Claim 4.3. We remark that the proof for $F$-factors (Theorem 1.7) is simpler than that of Hamilton cycles (Theorem 1.5). Indeed certain technicalities that arise for Hamilton cycles (e.g. the sets $Z_{1}$ and $Z_{2}$ and the constant $\alpha$ ) can be ignored. The reader may therefore prefer to concentrate just on $F$-factors on first read.

Proofs of Theorem 1.7 and 1.5 . Let $k$ and $c$ be given as in the theorems. For Theorem 1.7 we further have the parameter $f \geq k$. Let $\Delta=40$ and for convenience choose $\gamma \ll \beta \ll \alpha \ll 1 / \Delta, 1 / f$. 
We may also assume that $c \ll \gamma$ since it only appears in lower bounds. Apply Lemma 2.3 several times, each time with parameters $k, \Delta$, and $\overline{2.3}=c^{\prime} \ll c$, and for each $\int_{2.3} \in\left\{9 k^{2}-24 k+16, f^{2}-\right.$ $f\}$, and each $r_{2.3} \in\{k-1, f\}$, to obtain $\varepsilon^{\prime}$, the minimum 92.3 over all choices of $f 2.3$ and $r[2.3$. Choose $\varepsilon \ll \varepsilon^{\prime}$ and $n_{0}$ sufficiently large. Thus we may assume the following hierarchy of constants

$$
1 / n_{0} \ll \varepsilon \ll \varepsilon^{\prime} \ll c^{\prime} \ll c \ll \gamma \ll \beta \ll \alpha \ll 1 / \Delta, 1 / f .
$$

For Theorem 1.7 let a linear $k$-graph $F$ on $f$ vertices be given. We define

$\ell_{F}:=\operatorname{degen}(F)+\max _{e \in E(F)} \sum_{v \in e} \operatorname{deg}_{F}(v)=\operatorname{degen}(F)+\Delta^{\prime}(F)+k \quad$ and $\quad \ell_{\text {ham }}=\ell_{\text {ham }}(k):=k-1$

We apply Lemma 3.1 to obtain an absorber $\left(A_{F}, \mathcal{X}_{F}\right)$ of $F$ on $f^{2}$ vertices, of which $f$ are root vertices such that $A_{F}$ has degeneracy $\operatorname{degen}\left(A_{F}\right)=\ell_{F}$. Similarly, apply Lemma 3.2 to obtain a path absorber $\left(A_{\text {ham }}, \mathcal{X}_{\text {ham }}, y_{1}, y_{2}\right)$ on $9 k^{2}-23 k+15$ vertices, of which $k-1$ are roots such that $A_{\text {ham }}$ has degeneracy $\ell_{\text {ham }}$.

Suppose that $H$ is a $\left(p, \varepsilon p^{\ell}, \varepsilon\right)$-pseudo-random $k$-graph where $\ell=\ell_{F}$ when dealing with Theorem 1.7 and $\ell=\ell_{\text {ham }}$ when dealing with Theorem 1.5. We start with the following observation which will be used to include small sets of vertices in substructures of our desired spanning structure.

Claim 4.1. Suppose that $\hat{B}, \hat{X} \subset V(H)$ are disjoint sets, $\left(\hat{b}_{1}, \ldots, \hat{b}_{t}\right)$ is an ordering of the vertices of $\hat{B},|\hat{X}| \geq \max \left\{\gamma n / 4,800\left(f^{2}+9 k^{2}\right)^{2}|\hat{B}|\right\}$ and $\operatorname{deg}(\hat{b} ; \hat{X}) \geq c p|\hat{X}|^{k-1}$ for each $\hat{b} \in \hat{B}$. Then there is a set $\hat{B} \subseteq \hat{R} \subseteq \hat{B} \cup \hat{X}$ such that the following holds:

$F$-factors: If $\ell=\ell_{F}$, then $H[\hat{R}]$ contains an $F$-factor and $|\hat{R}| \leq f|\hat{B}|+f^{2}$.

Hamcyc: If $\ell=\ell_{\text {ham }}$ then $|\hat{R}|=3(t-1)(k-1)+1$ and $H[\hat{R}]$ contains a loose spanning path with end vertices $\hat{b}_{1}$ and $\hat{b}_{t}$ and such that $\hat{b}_{1}, \ldots, \hat{b}_{t}$ appear in this order in the path.

Proof. By Fact 1.8 we know that $H[\hat{B} \cup \hat{X}]$ is $\left(p, \varepsilon^{\prime} p^{\ell}, \varepsilon\right)$-pseudo-random. In particular, there are $f-1$ vertices $v_{1}, \ldots, v_{f-1} \in \hat{X}$ with $\operatorname{deg}\left(v_{i} ; \hat{X}\right) \geq \frac{p}{2}|\hat{X}|^{k-1}, i \in[f-1]$, and in the case of $F$ factors we may add some (at most $f-1$ ) of them to $\hat{B}$ so that $|\hat{B}|$ is a multiple of $f$. Abusing notation slightly we denote this modified set by $\hat{B}$. In the Hamilton cycle case we leave $\left(\hat{b}_{1}, \ldots, \hat{b}_{t}\right)$ unchanged.

In both cases, $\ell=\ell_{F}$ and $\ell=\ell_{\text {ham }}$, we apply Lemma 2.3 with $H_{[2.3}=H[\hat{B} \cup \hat{X}]$ and $Y_{[2.3}=\hat{B}$.

- If $\ell=\ell_{F}$ then we choose $8\left(A_{2.3}, \mathcal{X}_{2.3}\right)=\left(A_{F}, \mathcal{X}_{F}\right)$ and we fix $T[2.3$ as an $f$-uniform perfect matching on $\hat{B}$. The lemma then implies that there is a $T \sqrt{2.3}$-compatible family $\mathcal{A}_{F}$ of copies of $\left(A_{2.3}, \lambda(2.3)\right.$, i.e., a family of disjoint copies of $A_{F}$. Taking the complete $F$-factor in each of these copies yields an $F$-factor of $H[\hat{R}]=H\left[V\left(\mathcal{A}_{F}\right)\right]$ and certainly $\hat{B} \subset V\left(\mathcal{A}_{F}\right)$.

- If $\ell=\ell_{\text {ham }}$ then we let $T\left[2.3\right.$ be the 2 -uniform path with the vertex ordering $\hat{b}_{1}, \hat{b}_{2}, \ldots, \hat{b}_{t}$, i.e., $\hat{b}_{i} \hat{b}_{i+1} \in E(T / \sqrt{2.3})$ for all $i \in[t-1]$ and we let $\left(A^{\prime}, \mathcal{X}^{\prime}\right)$ be the loose path of length 9 three with the ends being the root vertices. Note that $\left(A^{\prime}, \mathcal{X}^{\prime}\right)$ has edge degeneracy two, thus at most $\ell_{\text {ham }}$. Applying Lemma 2.3 with $\left(A_{2.3}, \mathcal{X}_{2.3}\right)=\left(A^{\prime}, \mathcal{X}^{\prime}\right)$, we obtain a family $\mathcal{A}^{\prime}$ which consists of $t-1$ length three loose paths with ends $\hat{b}_{i}$ and $\hat{b}_{i+1}, i \in[t-1]$, and which are disjoint otherwise. Thus, $\hat{R}=V\left(\mathcal{A}^{\prime}\right)$ has size $3(t-1)(k-1)+1$ and $\mathcal{A}^{\prime}$ forms a spanning loose path with ends $\hat{b}_{1}$ and $\hat{b}_{t}$ in $H[\hat{R}]$, as required.

With this auxiliary claim proven we now turn to the construction of the absorbing set.

\footnotetext{
${ }^{8}$ One could equally just cover with copies of $F$, avoiding the use of absorbers here but we prefer to use absorbers for brevity.

${ }^{9}$ The length of a path is the number of edges in the path.
} 
Claim 4.2. Fix $m:=\lceil\beta n\rceil$. There are vertex sets $Z_{1} \cup Z_{2}=Z \subset A \subset V(H)$ and $U \subset V(H)$ of sizes $\left|Z_{1}\right|=m+\lceil\gamma n\rceil$ and $\left|Z_{2}\right|=m-\lceil\gamma n\rceil,|A \cup U| \leq 8 f^{2} \beta n$ such that

$$
\operatorname{deg}\left(v ; Z_{i}\right)>\frac{p}{4}\left|Z_{i}\right|^{k-1} \quad \text { for } i=1,2 \text { and any } v \in V \backslash U
$$

and such that $Z \subset A \cup U$ satisfies the following flexibility property:

$F$-factors: If $\ell=\ell_{F}$ then for any $Z^{\prime} \subset Z$ of size $m$ the subgraph $H\left[(A \cup U) \backslash Z^{\prime}\right]$ contains an $F$-factor.

Hamcyc: If $\ell=\ell_{\text {ham }}$ then there are $a_{1}, a_{2} \in A \backslash Z$ such that for any $Z^{\prime} \subset Z$ of size $m$ the subgraph $H\left[(A \cup U) \backslash Z^{\prime}\right]$ contains a spanning loose path with ends $a_{1}$ and $a_{2}$.

Proof. As explained in the beginning of this section we need to do some preprocessing as to guarantee (4.1). Let $r=f$ in the case of $F$-factors and $r=k-1$ in the case of finding a Hamilton cycle. Let $s=\left\lceil 10 k f \gamma^{-k} \varepsilon p^{\ell} n\right\rceil$. We choose disjoint sets $\hat{Z}_{1}, \hat{Z}_{2} \subset V$ of size $\left|\hat{Z}_{1}\right|=m+\lceil\gamma n\rceil+s$, $\left|\hat{Z}_{2}\right|=m-\lceil\gamma n\rceil+s$ and extend $\hat{Z}=\hat{Z}_{1} \cup \hat{Z}_{2}$ to a set $\hat{Y}_{0}$ of size $4 m+3 s$. Further, we choose disjoint sets $\hat{Y}_{1}, \ldots, \hat{Y}_{r-1} \subset V(H) \backslash \hat{Y}_{0}$ each of size $3 m+s$. Let $\hat{Y}=\hat{Y}_{0} \cup \cdots \cup \hat{Y}_{r-1}$, let $W \subset V \backslash \hat{Y}$ be a set of size $\alpha n$ and let

$$
B=\left\{v \in V: \operatorname{deg}(v ; S)<\frac{p}{2}|S|^{k-1}, \text { for some } S \in\left\{\hat{Z}_{1}, \hat{Z}_{2}, W, V \backslash(W \cup \hat{Y})\right\}\right\} .
$$

From the pseudo-randomness of $H$ we infer that $|B| \leq \varepsilon \gamma^{-(k-1)} p^{\ell} n$ and thus

$$
\operatorname{deg}(v ; V \backslash B) \geq \operatorname{deg}(v)-|B| n^{k-2}>\frac{c}{2} p n^{k-1} \quad \text { for each } v \in V .
$$

$F$-factors: For $\ell=\ell_{F}$ an application of Claim 4.1 with $\hat{B}=B, \hat{X}=V \backslash B$ then yields a set $U=\hat{R} \supset B$ of size $|U| \leq f(|B|+f)<s$ such that $H[U]$ contains an $F$-factor.

Hamcyc: For $\ell=\ell_{\text {ham }}$ we choose $u_{0}, v_{0} \in V \backslash B$ and let $\left(u_{0}, b_{1}, \ldots, b_{|B|}, v_{0}\right)$ be an ordering of the vertices of $B \cup\left\{u_{0}, v_{0}\right\}$. With this ordering and $\hat{X}=V \backslash\left(B \cup\left\{u_{0}, v_{0}\right\}\right)$ an application of Claim 4.1 then yields a set $U=\hat{R} \supset B$ of size $|U| \leq 3 k|B|<s$ such that $H[U]$ contains a spanning path with ends $u_{0}$ and $v_{0}$.

Consequently, from each of the sets $\hat{Z}_{1}, \hat{Z}_{2}, \hat{Y}_{0} \backslash \hat{Z}, \hat{Y}_{1}, \ldots, \hat{Y}_{r-1}$, we can remove a subset of size exactly $s$ to obtain sets $Z_{1} \cup Z_{2}=Z \subset Y_{0}, Y_{1}, \ldots Y_{r-1}$ with $\left|Z_{1}\right|=m+\lceil\gamma n\rceil,\left|Z_{2}\right|=m-\lceil\gamma n\rceil$, $\left|Y_{0}\right|=4 m,\left|Y_{1}\right|=\cdots=\left|Y_{r-1}\right|=3 m$, all disjoint from $U$. Let $Y=Y_{0} \cup \cdots \cup Y_{r-1}$, let $V^{\prime}=V \backslash U$. By the definition of $B$, the fact that $B \subset U$ and noting that $s \cdot n^{k-2}<\frac{p}{4}(\gamma n)^{k-1}$ we have that

$$
\operatorname{deg}(v ; S)>\frac{p}{4}|S|^{k-1} \text { for any } S \in\left\{Z_{1}, Z_{2}, W, V^{\prime} \backslash(W \cup Y)\right\} \quad \text { and any } \quad v \in V^{\prime} \cup\left\{u_{0}, v_{0}\right\} .
$$

In particular, (4.1) holds and we can now turn to the core of the proof of the claim.

Recall that $r=f$ in the case of $F$-factors and $r=k-1$ in Hamilton cycle case. In both cases we first apply Lemma 3.4 to obtain an $(r, m)$-template $T_{r}$ with vertex set $Y=Y_{0} \cup \cdots \cup Y_{r-1}$, maximum degree $\Delta_{1}\left(T_{r}\right) \leq \Delta$ and with the flexible set $Z \subset Y_{0}$. In particular, there is a perfect matching $M\left(Z^{\prime}\right)$ of $T_{r}\left[Y \backslash Z^{\prime}\right]$ for each set $Z^{\prime} \subset Z$ of size $m$. Then, apply Lemma 2.3 with $H_{[2.3}=H\left[V^{\prime} \backslash W\right], Y\left[2.3=Y, T\left[2.3=T_{r}\right.\right.$ and with $\left(A_{2.3}, \mathcal{X}_{2.3}\right)=\left(A_{F}, \mathcal{X}_{F}\right)$ in the case of $F$ factors, and with $(A 2.3, \mathcal{2 . 3})=\left(A_{\text {ham }}, \mathcal{X}_{\text {ham }}\right)$ in the case of Hamilton cycle. Note that all the conditions of Lemma 2.3 are indeed satisfied. In particular, the degeneracy of $(A[2.3, \overline{2.3})$ is at most $\ell$ (by our choice of $\ell_{F}$ and $\ell_{\text {ham }}$ ) and the maximum 2-degree condition in Lemma 2.3 is void for us here as $\ell \geq k-1$. This yields a $T_{f}$-compatible family $\mathcal{A}_{F}=\left\{\left(A_{e}, e\right)\right\}_{e \in E\left(T_{f}\right)}$ of copies of $\left(A_{F}, \mathcal{X}_{F}\right)$ with $Y \subset V(\mathcal{A}) \subset V^{\prime} \backslash W$ in the first case and a $T_{k-1}$-compatible family $\left.\mathcal{A}_{\text {ham }}=\left\{\left(A_{e}, e, u_{e}, v_{e}\right)\right\}_{e \in E\left(T_{k-1}\right)}\right]$ of copies of $\left(A_{\text {ham }}, \mathcal{X}_{\text {ham }}, y_{1}, y_{2}\right)$ with $Y \subset V\left(\mathcal{A}_{\text {ham }}\right) \subset V^{\prime} \backslash W$ in

\footnotetext{
${ }^{10}$ Recall that $u_{e}, v_{e} \notin e$ and thus they are distinct for all $e \in E\left(T_{k-1}\right)$.
} 
the second. In particular, by the defining property of $\left(A_{F}, \mathcal{X}_{F}\right)$ we infer that in any $\left(A_{e}, e\right) \in \mathcal{A}_{F}$ there is a complete $F$-factor, which covers all of $V\left(A_{e}\right)$, and an internal $F$-factor, which covers $V\left(A_{e}\right) \backslash e$. Similarly, in $\left(A_{e}, e, u_{e}, v_{e}\right) \in \mathcal{A}_{\text {ham }}$ there is a complete loose path, which covers $V\left(A_{e}\right)$ and an internal loose path, which covers $V\left(A_{e}\right) \backslash e$, both with the same end vertices $u_{e}$ and $v_{e}$. Moreover, being $T_{r}$-compatible any two copies $A_{e}$ and $A_{e^{\prime}}$ in $\mathcal{A}_{F}$ (in $\mathcal{A}_{\text {ham }}$, respectively) are disjoint if $e$ and $e^{\prime}$ are.

Together with the flexibility of $Z \subset Y$ with respect to the template $T_{f}$ we now easily establish the flexibility of $Z \subset V\left(\mathcal{A}_{F}\right) \cup U$, which thus concludes the proof for the case of $F$-factors by setting $A=V\left(\mathcal{A}_{F}\right)$. Indeed, let $Z^{\prime} \subset Z$ of size $m$ be given and let $M\left(Z^{\prime}\right) \subset E\left(T_{f}\right)$ denote a perfect matching of $Y \backslash Z^{\prime} \subset A$. By taking a complete $F$-factor of $A_{e}$ if $e \in M\left(Z^{\prime}\right)$ while taking an internal $F$-factor of $A_{e}$ if $e \in E\left(T_{f}\right) \backslash M\left(Z^{\prime}\right)$ we obtain an $F$-factor of $H\left[A \backslash Z^{\prime}\right]$. Together with the $F$-factor of $H[U]$ and $A \cap U=\emptyset$ this yields an $F$-factor of $H\left[(A \cup U) \backslash Z^{\prime}\right]$, as required.

By the same argument we obtain in the Hamilton cycle case that for any set $Z^{\prime} \subset Z$ of size $m$ there is a collection of $e\left(T_{k-1}\right)+1$ loose paths with fixed end vertices independent of $Z^{\prime}$, and which span the vertices of $H\left[\left(V\left(\mathcal{A}_{\text {ham }}\right) \cup U\right) \backslash Z^{\prime}\right]$. Indeed, this follows by considering a perfect matching $M\left(Z^{\prime}\right)$ of $T_{k-1}\left[Y \backslash Z^{\prime}\right]$ for a given set $Z^{\prime} \subset Z$ of size $m$ and taking in each $\left(A_{e}, e, u_{e}, v_{e}\right) \in \mathcal{A}_{\text {ham }}$ the complete path if $e \in M\left(Z^{\prime}\right)$ and the internal path if $e \in E\left(T_{k-1}\right) \backslash M\left(Z^{\prime}\right)$, both having end vertices $u_{e}$ and $v_{e}$. Together with the loose spanning path in $H[U]$ with end vertices $u_{0}$ and $v_{0}$ we obtain the required collection of $e\left(T_{k-1}\right)+1$ loose paths. Thus, to obtain $A$ and the flexibility of $Z \subset A \cup U$ it is left to connect the end vertices of these paths to obtain a long path.

Let $\left(A_{e_{1}}, \ldots, A_{e_{t}}\right)$ be an ordering of the elements of $\mathcal{A}_{\text {ham }}$. As the end vertices $u_{i}:=u_{e_{i}}$ and $v_{i}:=v_{e_{i}}$ of $A_{e_{i}}, i \in[t]$, are all contained in $V^{\prime}$ we can make use of (4.2) (with $S=W$ ) to apply Claim 4.1 with the ordering $\left(\hat{b}_{1}, \hat{b}_{2}, \ldots, \hat{b}_{2 t}\right)=\left(v_{0}, u_{1}, v_{1}, u_{2}, v_{2}, \ldots, u_{t}\right)$, where $t<\Delta_{1}\left(T_{k-1}\right) \cdot\left|Y_{1}\right| \leq$ $3 \Delta m$ and $\hat{X}=W$ to find a loose path connecting these vertices as given in the order. From this path we only keep the connecting paths $P_{i+1}$ between $v_{i}$ and $u_{i+1}, i=0, \ldots, t-1$, discarding each of the loose paths between $u_{i}$ and $v_{i}$. Now let $A=V\left(\mathcal{A}_{\text {ham }}\right) \cup V\left(P_{1}\right) \cup \cdots \cup V\left(P_{t}\right)$ and let $a_{1}=u_{0}$ and $a_{2}=v_{t}$. Together with the argument from above we conclude that $Z \subset A \cup U$ has the desired flexibility property.

Using Claim 4.2, we can now prove the following claim which will conclude the proof.

Claim 4.3. Let $V^{\prime \prime}=V \backslash(A \cup U)$ then there is a set $Z^{\prime} \subset Z$ of size $m$ such that the following holds:

$F$-factors: If $\ell=\ell_{F}$ then $H\left[V^{\prime \prime} \cup Z^{\prime}\right]$ contains an $F$-factor.

Hamcyc: If $\ell=\ell_{\text {ham }}$ then $H\left[V^{\prime \prime} \cup Z^{\prime} \cup\left\{a_{1}, a_{2}\right\}\right]$ contains a spanning loose path with ends $a_{1}$ and $a_{2}$.

Before proving the claim note that it implies the theorems. Indeed, if $\ell=\ell_{F}$ then we use Claim 4.3 and choose an $F$-factor of $H\left[V^{\prime \prime} \cup Z^{\prime}\right]$, for some $Z^{\prime} \subset Z$ of size $m$. Claim 4.2 then guarantees that there is an $F$-factor of $H\left[(A \cup U) \backslash Z^{\prime}\right]$ which thus yields an $F$-factor of $H$, as $V=V^{\prime \prime} \cup A \cup U$. For $\ell=\ell_{\text {ham }}$ we take a loose Hamilton path of $H\left[V^{\prime \prime} \cup Z^{\prime} \cup\left\{a_{1}, a_{2}\right\}\right]$ with ends $a_{1}$ and $a_{2}$. By Claim 4.2 there is a loose Hamilton path of $H\left[(A \cup U) \backslash Z^{\prime}\right]$ with the same end vertices which thus yields a Hamilton cycle of $H$.

Proof of Claim 4.3. Consider first the $F$-factor case. Let $R \subset V^{\prime \prime}$ be the largest set such that $H[R]$ contains an $F$-factor and let $L=V^{\prime \prime} \backslash R$ be the set of uncovered vertices. Suppose $|L| \geq \gamma n$, then there is a vertex $v \in L$ with $\operatorname{deg}(v ; L)>\frac{p}{2}|L|^{k-1}$ and by applying Claim 4.1 with $\hat{B}=\{v\}$ and $\hat{X}=L \backslash\{v\}$ we find a copy of $F$ in $L$, contradicting the maximality of $R$. Thus $|L|<\gamma n$ and the claim follows (by setting $R \cup S=V^{\prime \prime} \cup Z^{\prime}$ ) once we have shown that there is a set $S$ such that

(1) $L \subset S \subset L \cup Z$ and $H[S]$ contains an $F$-factor, 
(2) $Z \backslash S$ has size $m$.

To find $S$ consider first a smallest set $S_{1}$ which satisfies the first property. Such a set exists since we can apply Claim 4.1 with $\hat{B}=L$ and $\hat{X}=Z$, noting that the assumptions are met due to (4.1) and the fact that $L \subset V \backslash U$. Thus $S_{1}$ exists and $\left|S_{1}\right| \leq f(|L|+f)<m$. Now let $S_{2} \subset Z \backslash S_{1}$ be the largest set such that $H\left[S_{2}\right]$ contains an $F$-factor. By the same argument as in the previous paragraph $\left|Z \backslash\left(S_{2} \cup S_{1}\right)\right|<\gamma n$. Finally, due to Claim 4.2 we have

$$
\left|V^{\prime \prime}\right|+m=|V|-(|A \cup U|-m) \in f \mathbb{N},
$$

and

$$
\left|V^{\prime \prime}\right|+2 m=\left|V^{\prime \prime} \cup Z\right|=|R|+\left|S_{1}\right|+\left|S_{2}\right|+\left|Z \backslash\left(S_{1} \cup S_{2}\right)\right| .
$$

This yields $\left|Z \backslash\left(S_{1} \cup S_{2}\right)\right|-m \in f \mathbb{N}$ and therefore we can remove copies of $F$ from $S_{2}$ to obtain $S_{1} \subset S \subset S_{1} \cup S_{2}$ with the required properties.

Let us now turn to considering the Hamilton loose path. Here the argument is very similar to the above but it is slightly more delicate as we have to connect the loose paths that we find to one. For this, we use the partition of $Z$ into $Z_{1} \cup Z_{2}$ as in Claim 4.2, and carry out the argument using only vertices from $Z_{1}$, reserving the vertices of $Z_{2}$ to connect the paths in the very last step. The details follow.

In $V^{\prime \prime}$ we choose the largest set $R \subset V^{\prime \prime}$ with the property that $H[R]$ contains a loose Hamilton path with one of its end vertices, say, $a \in R$ satisfying $\operatorname{deg}\left(a ; V^{\prime \prime} \backslash R\right)>2 c p n^{k-1}$. Let $L=V^{\prime \prime} \backslash R$ and suppose that $|L| \geq \gamma n$. Then there is a vertex $b \in L$ with $\operatorname{deg}(b ; L)>4 c p n^{k-1}$. Applying Claim 4.1 with $\hat{B}=\{a, b\}$ and with $\hat{X}=L \backslash\{b\}$ we then find a path of length three in $L \cup\{a\}$ connecting $a$ and $b$, which thus yields a contradiction to the maximality of $R$. Thus $|L|<\gamma n$. Next, we claim that there is a set $S$ such that

(1) $L \subset S \subset L \cup Z_{1}$ and $H[S]$ has a spanning subgraph consisting of two vertex disjoint loose paths,

(2) $|Z \backslash S|=m+12(k-1)-4$.

To find $S$ consider first a smallest set $S_{1}$ with $L \subset S_{1} \subset L \cup Z_{1}$ and a largest set $S_{2} \subset Z_{1} \backslash S_{1}$ such that $H\left[S_{1}\right]$ and $H\left[S_{2}\right]$ both contain a Hamilton path. Due to (4.1) and the fact that $L \subset V \backslash U$ we can apply Claim 4.1 with an arbitrary ordering of the vertices of $\hat{B}=L$ and $\hat{X}=Z_{1}$. This shows that $S_{1}$ exists and $\left|S_{1}\right| \leq 3 k|L|<m-12(k-1)$. Further, using the same argument which was used to find $R$ above, we have that $\left|Z_{1} \backslash S_{2}\right|<\gamma n$, thus $\left|Z \backslash\left(S_{1} \cup S_{2}\right)\right|<\gamma n+\left|Z_{2}\right| \leq m$. Note that $\left|S_{i}\right| \equiv 1 \bmod (k-1), i=1,2$ and the same holds for $|R|$ and also for $(|A \cup U|-m)$ due to Claim 4.2, With $|V| \in(k-1) \mathbb{N}$ this yields

$$
\left|V^{\prime \prime}\right|+2 m=|V|+m-(|A \cup U|-m) \equiv m-1 \quad \bmod (k-1)
$$

and moreover we have

$$
\left|V^{\prime \prime}\right|+2 m=\left|V^{\prime \prime} \cup Z\right|=|R|+\left|S_{1}\right|+\left|S_{2}\right|+\left|Z \backslash\left(S_{1} \cup S_{2}\right)\right| .
$$

This yields $\left|Z \backslash\left(S_{1} \cup S_{2}\right)\right| \equiv m-4 \bmod (k-1)$ and therefore by shortening the path in $S_{2}$ we can enlarge $\left|Z \backslash\left(S_{1} \cup S_{2}\right)\right|$ and thus choose a set $S_{1} \subset S \subset S_{1} \cup S_{2}$ with the required properties.

Finally, let $\left(b_{1}, b_{2}\right),\left(c_{1}, c_{2}\right)$ and $\left(d_{1}, d_{2}\right)$ denote the ends of a Hamilton path in $H[R]$ and the two paths in $H[S]$ which cover all of $S$. Note that these vertices are contained in $V \backslash U$. Hence, by (4.1) we can apply Claim 4.1 with $\left(a_{2}, b_{1}\right)$ and $\hat{X}=Z_{2}$ and find a set $R_{a_{2}, b_{1}} \subset Z_{2}$ of size $3(k-1)-1$ which connect $a_{2}$ and $b_{1}$ by a loose path. We repeat the argument with $\left(b_{2}, c_{1}\right)$ and $\hat{X}=Z_{2} \backslash R_{a_{2}, b_{1}}$ to find $3(k-1)-1$ vertices in $Z_{2} \backslash R_{a_{2}, b_{1}}$ to connect $b_{2}$ and $c_{1}$ and in the same manner connect $\left(b_{2}, c_{1}\right),\left(c_{2}, d_{1}\right)$ and $\left(d_{2}, a_{1}\right)$. This yields a loose path with ends $a_{1}$ and $a_{2}$ which covers all but $|Z \backslash S|-12(k-1)+4=m$ vertices of $V^{\prime \prime} \cup Z$, and the claim follows. 


\section{REFERENCES}

[1] E. Aigner-Horev, D. Conlon, H. Hàn, Y. Person, and M. Schacht, Quasirandomness in hypergraphs, Electr. J. Comb. 25 (2018), no. 3, P3.34. 11, 1

[2] P. Allen, J. Böttcher, H. Hàn, Y. Kohayakawa, and Y. Person, Powers of Hamilton cycles in pseudorandom graphs, Combinatorica 37 (2017), no. 4, 573-616. 11

[3] P. Allen, J. Böttcher, J. Skokan, and M. Stein, Powers of Hamilton cycles in pseudorandom graphs, Random

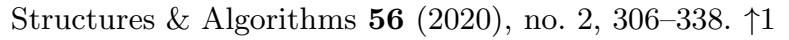

[4] P. Allen, J. Böttcher, H. Hàn, Y. Kohayakawa, and Y. Person, Blow-up lemmas for sparse graphs, arXiv preprint arXiv:1612.00622 (2016). 恛, 10

[5] N. Alon, Explicit Ramsey graphs and orthonormal labelings, Electron. J. Combin. 1 (1994), 12. 11010 1.2

[6] N. Alon and N. Kahale, Approximating the independence number via the $\vartheta$-function, Mathematical Programming 80 (1998), no. 3, 253-264. 11

[7] C. Berge, Graphs and hypergraphs, North-Holland Pub. Co., 1973. 11.1

[8] A. Bishnoi, F. Ihringer, and V. Pepe, A construction for clique-free pseudorandom graphs, Combinatorica (2020), 1-8. 佂

[9] E. Buß, H. Hàn, and M. Schacht, Minimum vertex degree conditions for loose Hamilton cycles in 3-uniform hypergraphs, J. Combin. Theory Ser. B 103 (2013), no. 6, 658-678. 13

[10] F. R. K. Chung, Quasi-random classes of hypergraphs, Random Structures \& Algorithms 1 (1990), no. 4, 363382. 佂

[11] Regularity lemmas for hypergraphs and quasi-randomness, Random Structures \& Algorithms 2 (1991), no. 2, 241-252. 1

[12] _ Quasi-random hypergraphs revisited, Random Structures \& Algorithms 40 (2012), no. 1, 39-48. 11

[13] F. R. K. Chung, R. L. Graham, and R. M. Wilson, Quasi-random graphs, Combinatorica 9 (1989), no. 4, 345362. 11 1

[14] F. R. K. Chung and R. L. Graham, Quasi-random hypergraphs, Random Structures \& Algorithms 1 (1990), no. 1, 105-124. 1 1

[15] D. Conlon, H. Hàn, Y. Person, and M. Schacht, Weak quasi-randomness for uniform hypergraphs, Random Structures \& Algorithms 40 (2012), no. 1, 1-38. 11 1

[16] D. Conlon, J. Fox, and Y. Zhao, Extremal results in sparse pseudorandom graphs, Advances in Mathematics 256 (2014), 206-290. 1

[17] _ A relative Szemerédi theorem, Geom. Funct. Anal. 25 (2015), no. 3, 733-762. 11

[18] P. Erdős and A. Hajnal, On Ramsey like theorems. Problems and results, Combinatorics (Proc. Conf. Combinatorial Math., Math. Inst., Oxford, 1972), 1972, pp. 123-140. MR0337636 11

[19] P. Erdős and J. Spencer, Imbalances in k-colorations, Networks 1 (1972), 379-385. 佔

[20] A. Ferber, G. Kronenberg, and K. Luh, Optimal threshold for a random graph to be 2-universal, Transactions of the American Mathematical Society 372 (2019), no. 6, 4239-4262. 13

[21] A. Ferber and R. Nenadov, Spanning universality in random graphs, Random Structures \& Algorithms 53 (2018), no. 4, 604-637. 13

[22] J. Friedman, Some graphs with small second eigenvalue, Combinatorica 15 (1995), no. 1, 31-42. 11 1, 1

[23] J. Friedman and A. Wigderson, On the second eigenvalue of hypergraphs, Combinatorica 15 (1995), no. 1, 43-65. 11, 1

[24] A. Frieze, M. Krivelevich, and P.-S. Loh, Packing tight Hamilton cycles in 3-uniform hypergraphs, Random Structures Algorithms 40 (2012), no. 3, 269-300. 11 1

[25] W. T. Gowers, Quasirandomness, counting and regularity for 3-uniform hypergraphs, Combin. Probab. Comput. 15 (2006), no. 1-2, 143-184. 11

[26] _ Hypergraph regularity and the multidimensional Szemerédi theorem, Ann. of Math. (2) 166 (2007), no. 3, 897-946. 11

[27] H. Hàn and M. Schacht, Dirac-type results for loose hamilton cycles in uniform hypergraphs, Journal of Combinatorial Theory (B) 100 (2010), no. 3, 332-346. 13

[28] J. Han, Y. Kohayakawa, P. Morris, and Y. Person, Clique-factors in sparse pseudorandom graphs, European Journal of Combinatorics 82 (2019), 102999. 1 3

[29] (2021), 87-108. 11 3

[30] J. Han, P. Morris, and A. Treglown, Tilings in randomly perturbed graphs: bridging the gap between HajnalSzemerédi and Johansson-Kahn-Vu, Random Structures \& Algorithms 58 (2021), no. 3, 480-516. 13

[31] J. Haviland and A. Thomason, Pseudo-random hypergraphs, 1989, pp. 255-278. Graph theory and combinatorics (Cambridge, 1988). 11 1 
[32] Y. Kohayakawa, B. Nagle, V. Rödl, and M. Schacht, Weak hypergraph regularity and linear hypergraphs, J. Combin. Theory Ser. B 100 (2010), no. 2, 151-160. 1, 1, 1, 2.1

[33] Y. Kohayakawa, V. Rödl, and P. Sissokho, Embedding graphs with bounded degree in sparse pseudorandom graphs, Israel Journal of Mathematics 139 (2004), 93-137. 11 1

[34] Y. Kohayakawa, V. Rödl, and J. Skokan, Hypergraphs, quasi-randomness, and conditions for regularity, J. Combin. Theory Ser. A 97 (2002), no. 2, 307-352. 1

[35] Y. Kohayakawa, G. O. Mota, M. Schacht, and A. Taraz, Counting results for sparse pseudorandom hypergraphs I, European J. Combin. 65 (2017), 276-287. 11

[36] - Counting results for sparse pseudorandom hypergraphs II, European J. Combin. 65 (2017), 288-301. 11

[37] Y. Kohayakawa, V. Rödl, M. Schacht, P. Sissokho, and J. Skokan, Turán's theorem for pseudo-random graphs, J. Combin. Theory Ser. A 114 (2007), no. 4, 631-657. 11

[38] J. Komlós, G. N. Sárközy, and E. Szemerédi, Blow-up lemma, Combinatorica 17 (1997), no. 1, 109-123. 11 1

[39] M. Krivelevich and B. Sudakov, Pseudo-random graphs, More sets, graphs and numbers, 2006, pp. 199-262. 11. 1 , 2.2

[40] M. Krivelevich, B. Sudakov, and T. Szabó, Triangle factors in sparse pseudo-random graphs, Combinatorica 24 (2004), no. 3, 403-426. 1

[41] M. Kwan, Almost all Steiner triple systems have perfect matchings, Proceedings of the London Mathematical Society 121 (2020), no. 6, 1468-1495. 13

[42] J. Lenz and D. Mubayi, Eigenvalues and linear quasirandom hypergraphs, Forum Math. Sigma 3 (2015), e2, 26. 11010 1

[43] J. Lenz and D. Mubayi, The poset of hypergraph quasirandomness, Random Structures \& Algorithms 46 (2015), no. $4,762-800$. 佂

[44] _ Perfect packings in quasirandom hypergraphs I, J. Comb. Theory Ser. B 119 (July 2016), no. C, $155-177$. 11, 1.1. 1, 1. 1.3.

[45] J. Lenz, D. Mubayi, and R. Mycroft, Hamilton cycles in quasirandom hypergraphs, Random Structures \& Algorithms 49 (2015). 怆 1, 1.2, 1, 3

[46] R. Montgomery, Embedding bounded degree spanning trees in random graphs., arXiv preprint arXiv:1405.6559 (2014). 11.1, $3,3.3$

[47] _ Spanning trees in random graphs, Advances in Mathematics 356 (2019), 106793. 11.1 33.3

[48] R. Nenadov, Triangle-factors in pseudorandom graphs, Bulletin of the London Mathematical Society 51 (2019), no. 3, 421-430. 11 1 1

[49] R. Nenadov and Y. Pehova, On a Ramsey-Turán variant of the Hajnal-Szemerédi theorem, SIAM Journal on Discrete Mathematics 34 (2020), no. 2, 1001-1010. 13

[50] V. Rödl and M. Schacht, Regular partitions of hypergraphs: counting lemmas, Combin. Probab. Comput. 16 (2007), no. 6, 887-901. 11

[51] Legular partitions of hypergraphs: regularity lemmas, Combin. Probab. Comput. 16 (2007), no. 6, 833885. 价

[52] V. Rödl and J. Skokan, Regularity lemma for k-uniform hypergraphs, Random Structures \& Algorithms 25 (2004), no. 1, 1-42. 1

[53] V. Rödl, A. Ruciński, and E. Szemerédi, A Dirac-type theorem for 3-uniform hypergraphs, Combinatorics, Probability and Computing 15 (2006), no. 1-2, 229-251. 11.1

[54] J. H. Spencer, Turán's theorem for k-graphs., Rand Res. 4648 (1971), no. 1. 11.1

[55] A. Thomason, Pseudorandom graphs, Random graphs '85 (Poznań, 1985), 1987, pp. 307-331. 11

[56] _ Random graphs, strongly regular graphs and pseudorandom graphs, Surveys in combinatorics 1987 (New Cross, 1987), 1987, pp. 173-195. 怆

[57] H. Towsner, $\sigma$-algebras for quasirandom hypergraphs, Random Structures \& Algorithms 50 (2017), no. 1, 114139. 怆 1 